\title{
Implementation of a New DTSTEP Algorithm for Use in RELAP5-3D and PVMEXEC Completion Report
}

George L. Mesina

December 2010

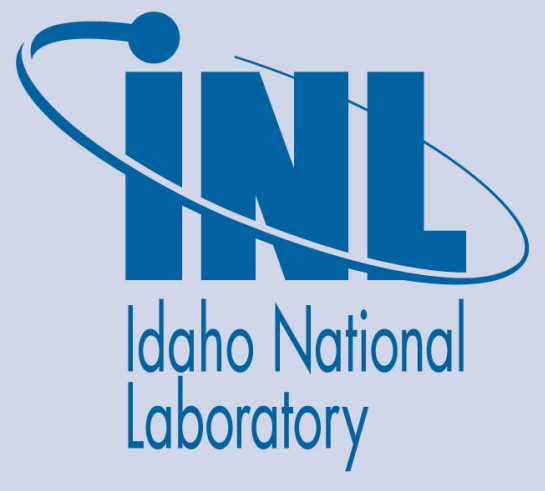

The INL is a U.S. Department of Energy National Laboratory operated by Battelle Energy Alliance 
INL/EXT-11-20798

\title{
Implementation of a New DTSTEP Algorithm for Use in RELAP5-3D and PVMEXEC Completion Report
}

\author{
George L. Mesina
}

December 2010

\begin{abstract}
Idaho National Laboratory
Idaho Falls, Idaho 83415
\end{abstract}

http://www.inl.gov

Prepared for the

U.S. Department of Energy

Office of Naval Reactors

Under DOE Idaho Operations Office

Contract DE-AC07-05ID14517 

Implementation of a New DTSTEP Algorithm
for use in RELAP5-3D and PVMEXEC
Page 1
Completion Report

INL/EXT-11-20798

\author{
Implementation of a New DTSTEP Algorithm \\ for use in RELAP5-3D and PVMEXEC \\ Completion Report
}

Dr. George L Mesina

December, 2010 

Implementation of a New DTSTEP Algorithm Page 2
for use in RELAP5-3D and PVMEXEC
Completion Report

INL/EXT-11-20798

\begin{abstract}
Implementation of a New DTSTEP Algorithm for use in RELAP5-3D and PVMEXEC

Completion Report
\end{abstract}

Dr. George L Mesina

December, 2010

\title{
Idaho National Laboratory Thermal Science and Safety Analysis Idaho Falls, Idaho 83415 http://www.inl.gov
}

\author{
Prepared for the \\ U.S. Department of Energy \\ Office of Naval Reactors \\ Under DOE Idaho Operations Office \\ Contract DE-AC07-05ID14517
}




\section{Implementation of a New DTSTEP Algorithm Page 3 for use in RELAP5-3D and PVMEXEC \\ Completion Report \\ Implementation of a New DTSTEP Algorithm \\ for use in RELAP5-3D and PVMEXEC \\ Completion Report}

INL/EXT-11-20798

December, 2010

Approved by:

Dr. George Mesina

Date

Author

Robert Martin

Technical Reviewer

Date

Hope Forsmann

Date

Technical Reviewer

Dr. James Wolf

Date

Project Manager

George Griffith

Date

Department Manager 


\title{
Implementation of a New DTSTEP Algorithm for use in RELAP5-3D and PVMEXEC
}

\author{
Completion Report
}

\section{EXECUTIVE SUMMARY}

The PVM Coupling methodology for decomposing a complex model into domains onto which individual programs may be applied has proven effective for solving many multi-physics problems. There have been, from the outset, some detailed and/or long-running models that cause the process to fail. Some 26 errors are listed in Tables 1 and 18 and in Section 11. These arise from deficiencies in the floating point calculation and testing of time steps, cumulative time, and time targets, along with unforeseen subtleties in the coupling technology.

This project addressed the PVM coupling issues surrounding the DTSTEP subroutines on RELAP53D and PVMEXEC. The algorithmic replacement of floating point control of these items with an integer based time-step method resolved these and related issues. This report documents the theory and implementation of the integer timestep methodology.

This report also provides a great deal of information about DTSTEP for code development and debugging. Alphabetized lists of all variables in both DTSTEP subroutines (RELAP5-3D and PVMEXEC) and related Fortran modules are organized into numerous tables according the subroutine or module in which it occurs. Many internal subprograms were also created in both DTSTEP subroutines and in some modules. Alphabetized lists of these subroutines and functions, with brief subprogram descriptions, are stored in other tables. 


\section{Implementation of a New DTSTEP Algorithm for use in RELAP5-3D and PVMEXEC}

\section{Completion Report}

\section{Checklist}

\begin{tabular}{|l|l|}
\hline Good & Originality \\
\hline Good & Scientific Relevance \\
\hline Excellent & Completeness \\
\hline Acceptable & Acknowledgement \\
\hline Good & Organization \\
\hline Excellent & Clarity of writing \\
\hline Checked & No mention of funding source \\
\hline Checked & $\begin{array}{l}\text { No naming of funding source staff except in references to } \\
\text { open literature }\end{array}$ \\
\hline Checked & $\begin{array}{l}\text { No mention of alternative names for RELAP5-3D or } \\
\text { PVMEXEC }\end{array}$ \\
\hline
\end{tabular}




\section{Implementation of a New DTSTEP Algorithm for use in RELAP5-3D and PVMEXEC}

\section{Completion Report}

\section{CONTENTS}

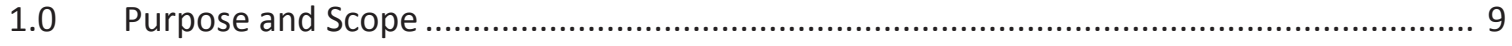

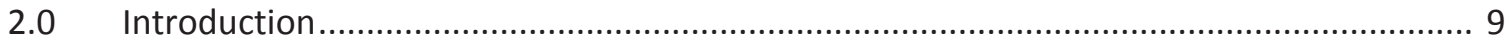

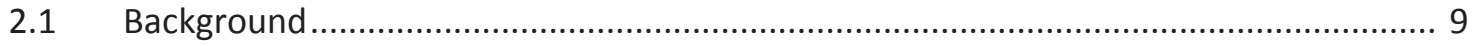

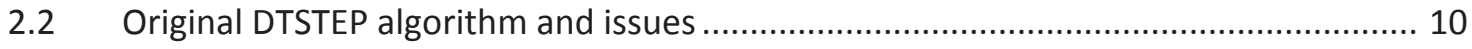

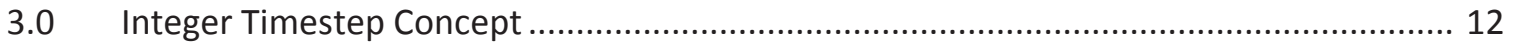

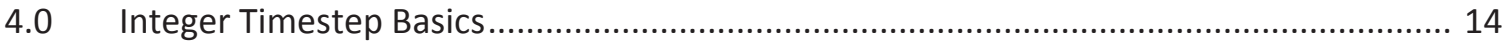

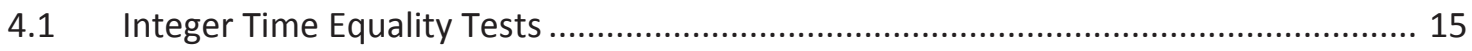

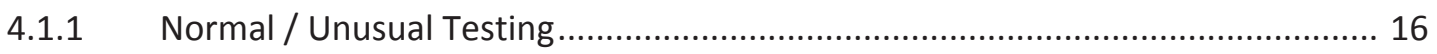

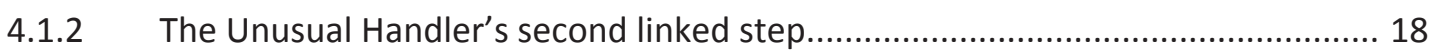

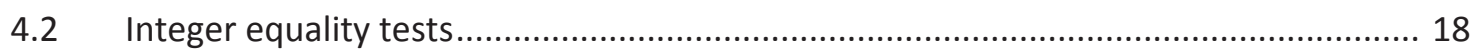

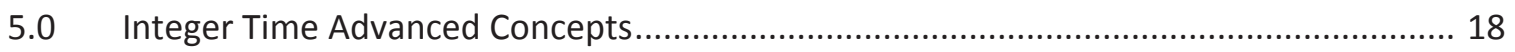

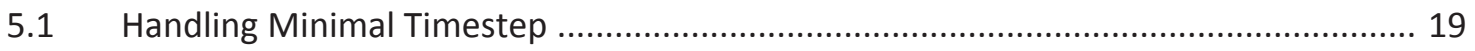

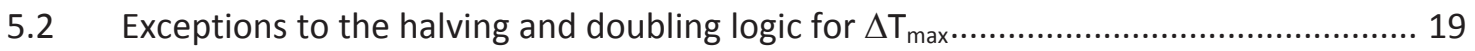

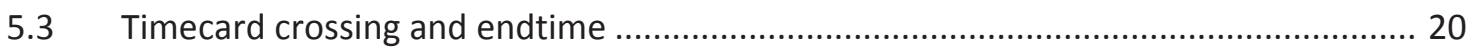

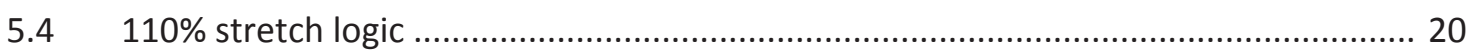

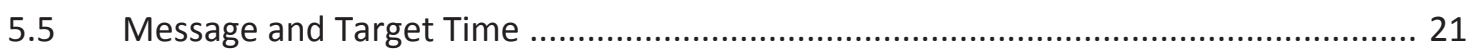

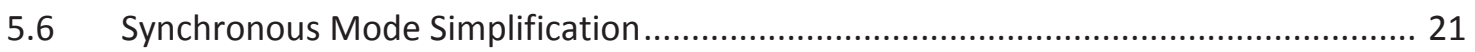

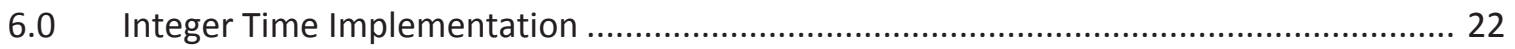

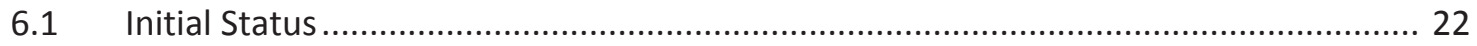

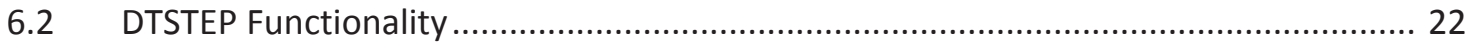

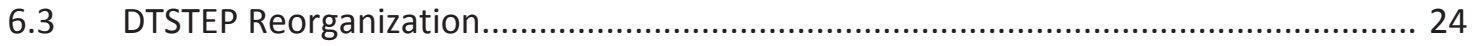

6.4 Define, mnemonically rename, eliminate variables, and fix declarations, create integer

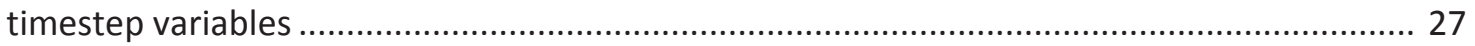

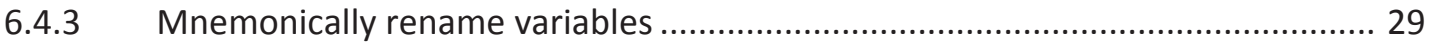

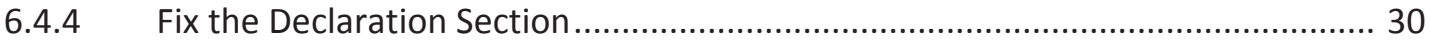

6.4.5 New Integer-Algorithm Variables ...................................................................... 30

6.5 New Integer Timestepping Modules ...................................................................... 31

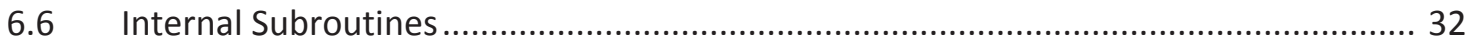

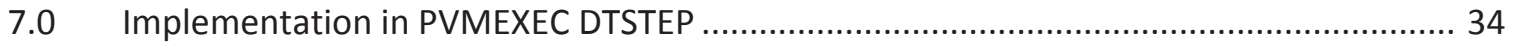

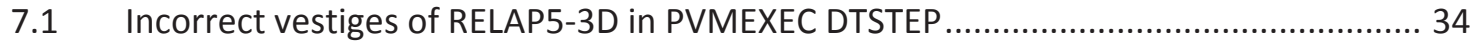

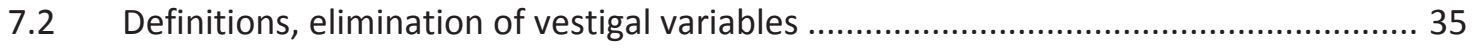

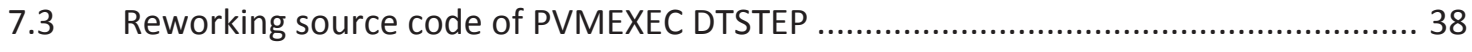

7.3.1 Deleted source code from PVMEXEC DTSTEP .................................................. 38 


\section{Implementation of a New DTSTEP Algorithm for use in RELAP5-3D and PVMEXEC}

Completion Report

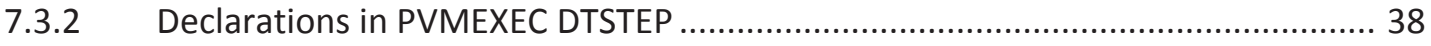

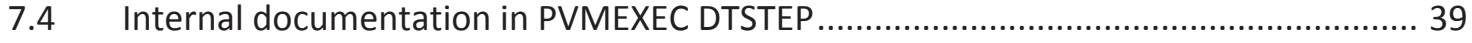

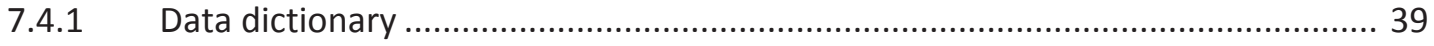

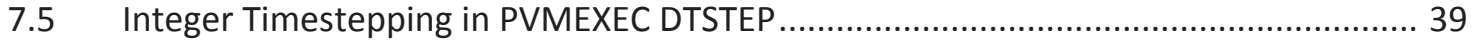

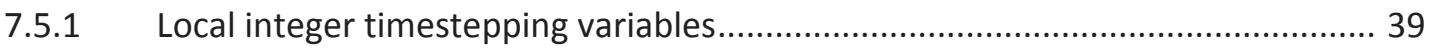

7.5.2 Module integer timestepping variables ........................................................... 40

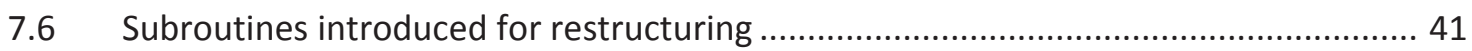

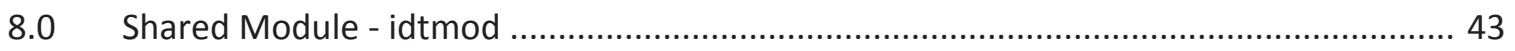

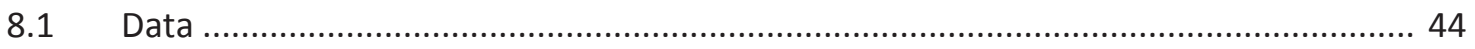

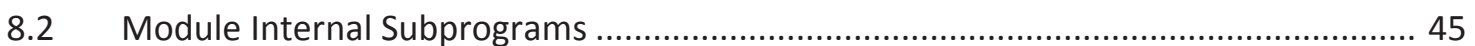

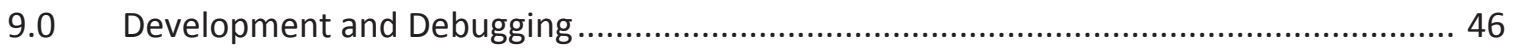

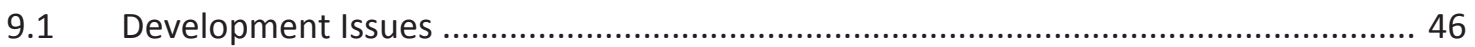

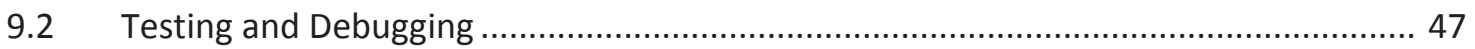

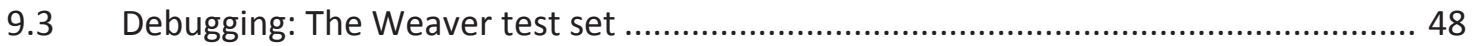

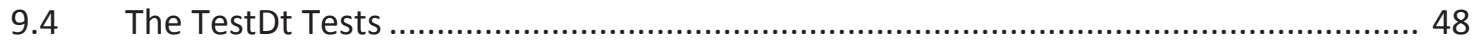

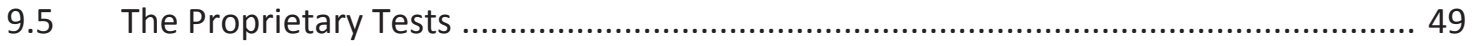

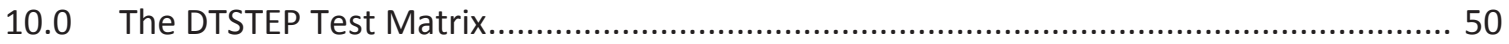

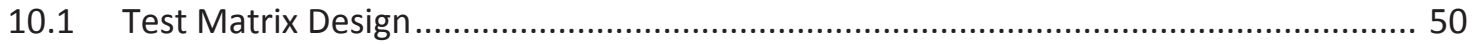

10.1.1 Package A Timestep Sizes (34 tests) .............................................................. 51

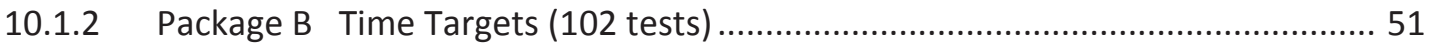

10.1.3 Package C Normal/Unusual Time (408 tests) .................................................. 52

10.1.4 Package D Coupling Configurations (2856 tests) .............................................. 52

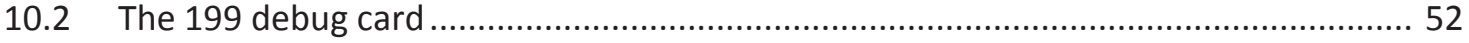

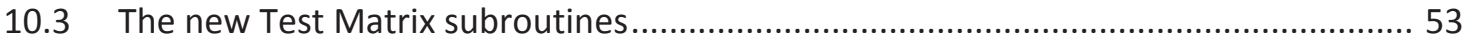

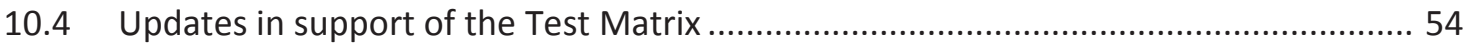

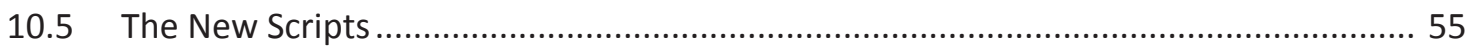

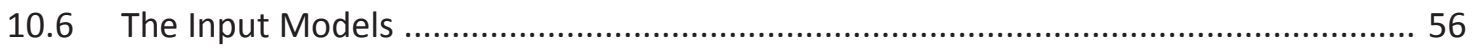

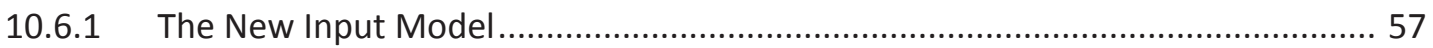

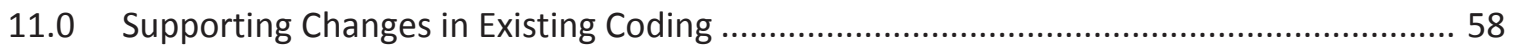

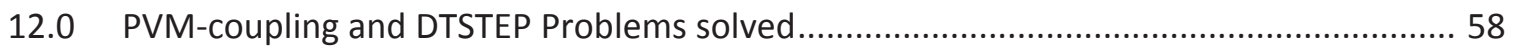

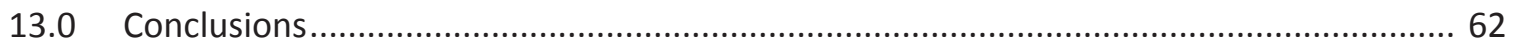

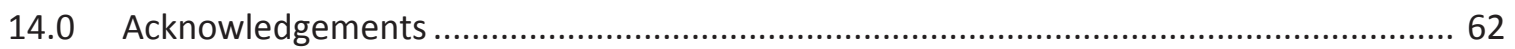

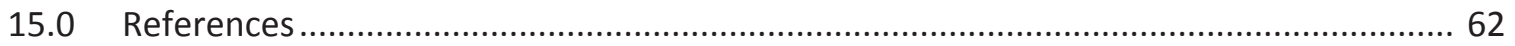

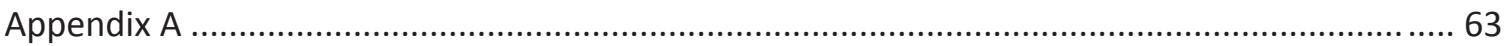




\title{
Implementation of a New DTSTEP Algorithm for use in RELAP5-3D and PVMEXEC
}

\author{
Completion Report
}

\section{Figures}

Figure 1. Pictorial presentation of tolerance test Type I and II errors ....................................... 12

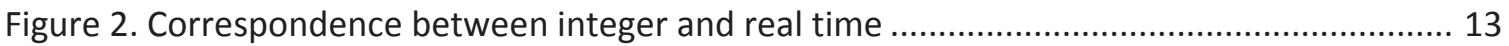

Figure 2a. Unusual times cannot be represented exactly by an integer..................................... 14

Figure 3a. First portion of "Sectional Flowchart" for RELAP5-3D DTSTEP Functions ..................... 25

Figure 3b. Second portion of "Sectional Flowchart" for RELAP5-3D DTSTEP Functions ................ 26

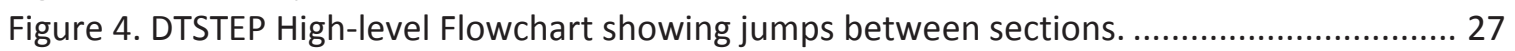

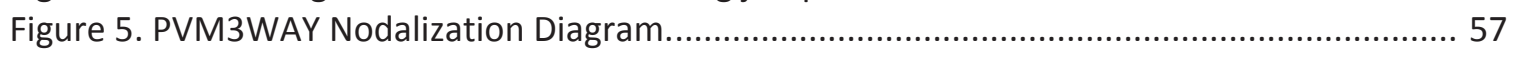

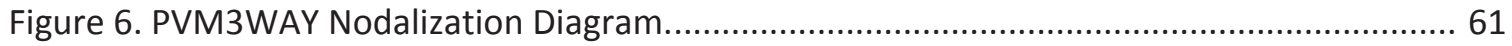

\section{Tables}

Table 1. Some User Problems associated with Coupling Calculations and DTSTEP ...................... 9

Table 2. Example of tolerance test "Type I Error" at a communication point ............................. 11

Table 3. Example of tolerance test "Type I Error" at a communication point .............................. 11

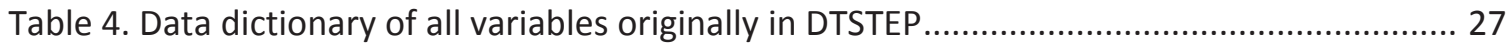

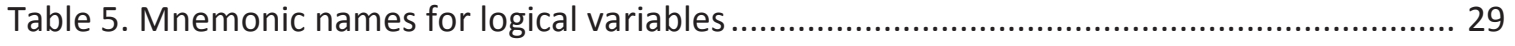

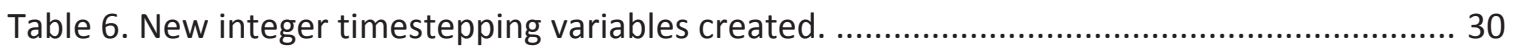

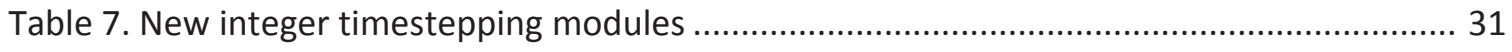

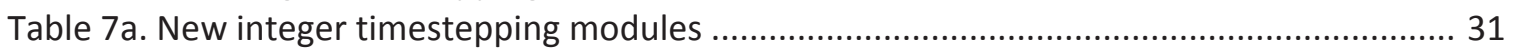

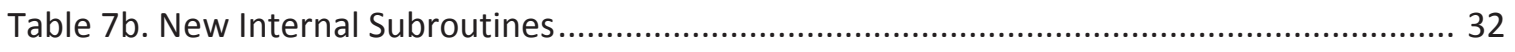

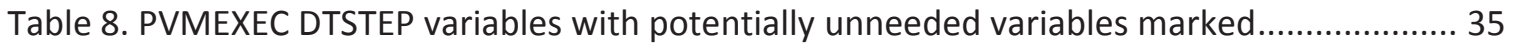

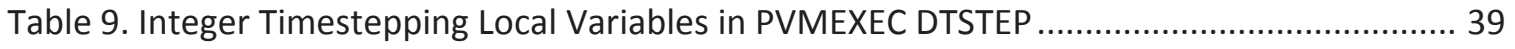

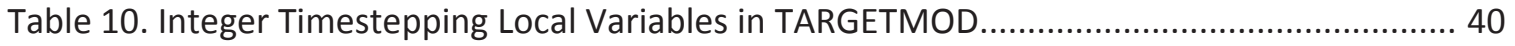

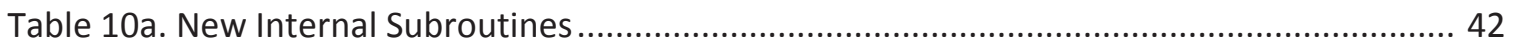

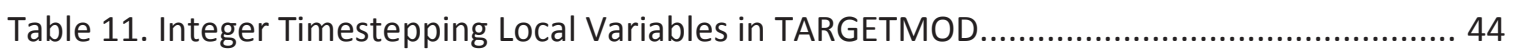

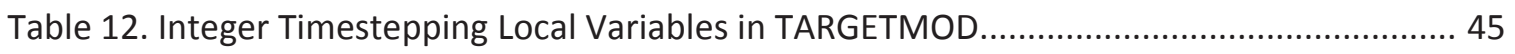

Table 13. TestDt input models for debugging unavailable input models .................................... 48

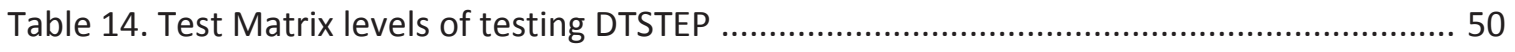

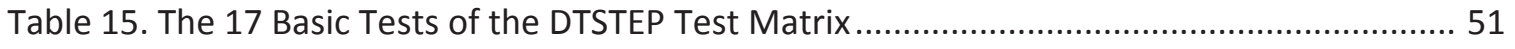

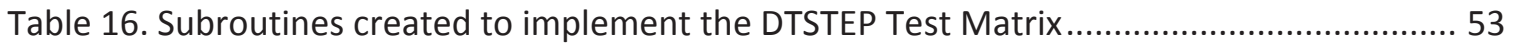

Table 17. Implementation of Test Matrix basic tests within RELAP5 DTSTEP...............................5 54

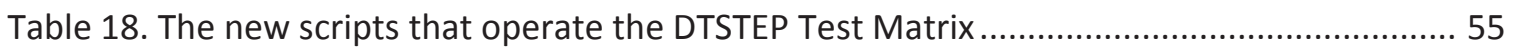

Table 19. Test Matrix base input tests, the models and their sets of input files .......................... 56 


\section{Implementation of a New DTSTEP Algorithm for use in RELAP5-3D and PVMEXEC}

\subsection{Purpose and Scope}

The purpose of this report is to document the changes to the timestep advancement and selection scheme in RELAP5-3D and PVMEXEC that were successfully implemented.

The algorithm development and work to implement the new algorithm under the constraints of the project is reported. This document covers developments and testing through released version r3d244b, developmental version prB2441b.

\subsection{Introduction}

\subsection{Background}

The PVM coupling capability was incorporated into RELAP5-3D beginning in 1999. Since then, several User Problems (UP) have been reported, including the sample appearing in Table 1.

The most common failure is by "hanging the machine." This means that processes participating in the coupled calculation have arrived at coding that waits for a particular message to arrive, but those messages have not been and cannot be sent. Thus no further progress can be made on the calculation. Another type of coupling calculation error is the failure to perform edits such as printed output, restart output, and even plot output. Some coupling-related errors include restarts that either fail or differ from the same transient run without restarting. Another class of errors involve problem that stop before the end of transient and those that do not stop at all.

Table 1. Some User Problems associated with Coupling Calculations and DTSTEP

\begin{tabular}{|l|l|}
\hline $\begin{array}{l}\text { UP } \\
\text { Number }\end{array}$ & Description \\
\hline 03009 & 6000 s transient doesn't stop. No plot, major, or restart from 3000 s onwards \\
\hline 03019 & Marviken deck gets no major or restart edit at end of transient \\
\hline 03022 & pvmedax.ii problem core dumps during transient \\
\hline 03024 & $\begin{array}{l}\text { VHTR calculation stops at } 1^{\text {st }} \text { timecard end } 28800 \text { s rather than } 2^{\text {nd }} \text { card's end time } \\
345600 \mathrm{~s}\end{array}$ \\
\hline 04011 & A 180 s transient w/ Dt=0.00005 (3600000 advancements) runs past end time. \\
\hline 05001 & A long-running calculation wrote no restart edits beyond a certain time. \\
\hline 06020 & Code stopped writing major edits after 277200 s for a VHTR deck. \\
\hline 06034 & RCCS pebble bed reactor calculation wrote no major edits at 10,050 or $45,000$. \\
\hline 08015 & A 3 loop system calculation the code hangs possibly due to velocity flip-flop. \\
\hline
\end{tabular}

In Table 1, the UP number has the form XXYYY, where XX are the last two digits of the year, and yyy is the number of the problem in the sequence they were submitted that year.

Not all of the UP listed in Table 1 were ever resolved. Many of those listed were solved by modifying the floating point calculations and/or tests. However, as examination of the descriptions will attest, the same kind of problem occurred later for another user input model. 


\title{
Implementation of a New DTSTEP Algorithm for use in RELAP5-3D and PVMEXEC
}

\author{
Completion Report
}

In more recent years, code hangs were encountered with more detailed models and often with long-running transients. These are recorded in Sec. 10.1 Table 18.

Permanent resolution of all these errors was the purpose of the project described herein. These errors are related to the timestepping algorithms embodied in the subroutines named DTSTEP in both RELAP5-3D and PVMEXEC, but primarily in RELAP5-3D.

\subsection{Original DTSTEP algorithm and issues}

The creation of PVMEXEC, Sec. 19 Ref. (a), and the modification of RELAP5-3D, Sec. 19 Ref. (b), to operate with PVMEXEC required a fundamental change to the timestep selection and advancement scheme. Originally, the user-selected timestep controls, $\Delta T_{\max }$ and $\Delta T_{\min }$, were the primary control mechanism. First, for all timesteps:

$$
\Delta T_{\text {max }} \geq \Delta t_{i} \geq \Delta T_{\min } \text { for every timestep i }
$$

Second, the algorithm enforces a halving/doubling timestep adjustment, subject to (1). The algorithm cuts the timestep in half and repeats the advancement from the previously successful time level when limits, such as mass-error upper bound or material Courant limit, are exceeded during the new advancement. Other conditions allow timestep doubling, but only after an even number of advancements with its current timestep. Therefore, with the exception of Rule (4) below, all timesteps have the form:

$$
\Delta t_{i}=\Delta T_{\max } / 2^{k}, \text { subject to (1) }
$$

Third, the cumulative time $T_{J}=\sum_{i=1}^{i=J} \Delta t_{i}$ attained every integer multiple of $\Delta \mathrm{T}_{\max }$ up to the final time, regardless of the timesteps taken. A mathematical expression of this is: For every integer $j$, there is a J for which:

$$
j \times \Delta T_{\max }=T_{J}\left(\equiv \sum_{i=1}^{i=J} \Delta t_{i}\right)
$$

Because floating point addition is inaccurate due to roundoff error, this rule was enforced by use of integer-based control. The singular exception to rules (2) and (3) was a timecard endtime, $\mathrm{E}$, a user-selected time target that is not a multiple of $\Delta \mathrm{T}_{\max }$. If a slightly larger timestep, up to $110 \%$ of $\Delta T_{\max }$, could reach the endtime, the necessary timestep was taken, even if it did not have form (1). Algorithmically,

$$
\text { if }\left(T_{i}+1.1 \Delta t_{i+1} \geq E\right) \text { then } \Delta t_{i+1}=E-T_{i}
$$

Because of rules (3) and (4), all RELAP5-3D user-requested output (major and minor edits, plots and restarts) is written only at multiples of $\Delta \mathrm{T}_{\max }$ or at timecard endtimes and there were no issues with output.

With the addition of coupling to RELAP5-3D, there is need to reach arbitrary communication points, $C_{M}$, that may violate rule (3) without being a timecard endtime. Resolution of this 


\section{Implementation of a New DTSTEP Algorithm for use in RELAP5-3D and PVMEXEC}

Completion Report

requirement could be handled three ways: a new algorithm, a generalization of the existing algorithm, or restrictions on user input. The latter was deemed unacceptable as it would require code users to ensure that the same timestep input was used in the PVMEXEC and RELAP5-3D input decks. A choice was made to generalize the existing timestep algorithm.

Inaccuracies in floating point arithmetic make reaching unusual communication time targets difficult if PVMEXEC and RELAP5-3D are on different compute platforms. This is caused by the well-known issue of accumulation of floating point round-off error. Table 2 shows that starting at the same time and taking the same sized steps results in different cumulative times on a 64bit floating-point Opteron vs. AMD 64 chip. Moreover, on a given processor, using a smaller timestep to arrive at the same target time $(1005 \mathrm{sec})$ produces different roundoff error.

Table 2. Example of tolerance test "Type I Error" at a communication point

\begin{tabular}{|l|l|l|}
\hline Specification & $\mathbf{T}_{\text {start }}=\mathbf{1 0 0 0 . 0}, \mathbf{N}=\mathbf{1 0 0}$ timesteps & $\Delta \mathbf{t}=\mathbf{0 . 0 0 5}$ \\
\hline Opteron & $\mathrm{T}=1005.0000000745$ & \\
\hline AMD 64 & $\mathrm{T}=1004.999999888$ & \\
\hline Specification & $\mathrm{T}_{\text {start }}=\mathbf{1 0 0 0 . 0}, \mathbf{N}=\underline{\mathbf{1 0 0 0}}$ timesteps & $\Delta \mathrm{t}=\underline{\mathbf{0 . 0 0 0 5}}$ \\
\hline AMD 64 & $\mathrm{T}=1005.000000238$ & \\
\hline
\end{tabular}

Since communication points, $C_{M}$, may not occur at multiples of $\Delta T_{M A X}$, the integer-based control for reaching output points could not apply and it is clear that a floating point equality test would fail due to roundoff. A standard computer science solution is to apply a floating-point tolerance test rather than an equality test. In generalizing the algorithm, all communication and output times were evaluated using tolerance tests. The test function and test form were:

$$
\begin{gathered}
C\left(T_{i}, \Delta t\right) \equiv\left|T_{i}+\Delta t-C_{M}\right| \\
\text { if }\left(C\left(T_{i}, \Delta t_{i+1}\right)<\varepsilon\right) \text { perform communication }
\end{gathered}
$$

Communication between RELAP5-3D and the PVMEXEC took place when cumulative time was sufficiently close to communication target, $C_{M}$. The tolerance, $\varepsilon$, was taken to be larger than the round-off error produced in the test problems, but small enough to force communication at the correct time; $\varepsilon=10^{-8}$ was used.

There were two opposing problems with the approach. The first problem occurs when round-off alters cumulative time by more than $\varepsilon$. In Table 2 , round-off is in excess of $2 \times 10^{-8}>\varepsilon$. Thus, a tolerance test of the form ( $5 \mathrm{a}$ ) can activate at the wrong time or can be entirely bypassed. For example, with $\mathrm{T}_{\text {start }}=1.0, \mathrm{C}_{\mathrm{M}}=1.001, \Delta \mathrm{T}_{\max }=10^{-2}, \Delta \mathrm{T}_{\min }=10^{-7}, \Delta \mathrm{t}_{\text {smallest }}=1.52587887214593 \times 10^{-7}$ (via repeated halving). If every timestep were at this smallest $\Delta t$, the results are in Table 3.

Table 3. Example of tolerance test "Type I Error" at a communication point

\begin{tabular}{|l|l|l|}
\hline Step \# & $\mathrm{T}_{\mathrm{i}}=$ Cumulative Time & $\mathrm{C}\left(\mathrm{T}_{\mathrm{i}}, \Delta \mathrm{t}\right)=\left|\mathrm{T}_{\mathrm{i}}+\Delta \mathrm{t}-\mathrm{C}_{\mathrm{M}}\right|$ \\
\hline 6553 & 1.0009990842492 & $1.38 \times 10^{-7}>\varepsilon$ \\
\hline 6554 & 1.0010000610128 & $1.43 \times 10^{-8}>\varepsilon$ \\
\hline
\end{tabular}




\section{Implementation of a New DTSTEP Algorithm for use in RELAP5-3D and PVMEXEC}

The difference is calculated according to Eq (5). Since both differences exceed $\varepsilon$, the test (5a) can never activate; as shown in Figure 1. Several User Problems, UP, were reported that had this at their source. In statistical terms, the null hypothesis is "test is true when $T_{i}$ is closest." This is always rejected, even when true (on step 6554). Rejecting the null hypothesis when it is true is called Type I error or a false positive. Type I error can be overcome by making $\varepsilon$ larger. For example, if $\varepsilon=2 \times 10^{-8}$, the test would activate on step 6554 . It could also be overcome by making $\Delta \mathrm{T}_{\min }$ smaller; this leads to a problem with type II error as discussed next.

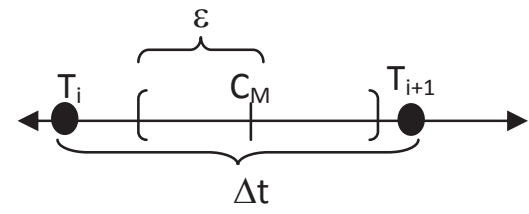

Type I Error: Bypass communication pt.

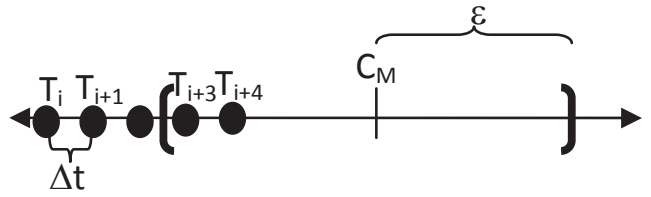

Type II Error: Activate before closest value

Figure 1. Pictorial presentation of tolerance test Type I and II errors

In fact, RELAP5-3D input allows $\Delta \mathrm{t}_{\mathrm{MIN}} \geq 10^{-12}$; so $\Delta \mathrm{T}_{\min }<\varepsilon=10^{-8}$ is allowed. With such input, tolerance tests like $(5 a)$ will activate on the very first time $T_{i}+\Delta t_{i+1}$ is within $\varepsilon=10^{-8}$ of $C_{M}$. As shown in Figure 1, this occurs before the intended activation time (the closest cumulative time). The test activates when it should not. Accepting the null hypothesis when it is false is called Type II error or a false negative. Type II error can be overcome by making s smaller.

It is clear that altering $\varepsilon$ can never overcome both types of error as Type I error requires $\varepsilon$ to be increased while Type II error requires $\varepsilon$ to be reduced. Nevertheless, attempts were made to determine a value of $\varepsilon$ that was large enough such that round-off error could be mitigated and small enough to preclude false negatives. Application of the coupled code system to complex and long running problems of interest revealed several failures in the floating point-based algorithm. Other slight modifications of the floating point timestep algorithm were introduced; failures were resolved, however, the general Type I and Type II error problems were not.

\subsection{Integer Timestep Concept}

Since the floating point tolerance test was insufficient to resolve the combined Type I and Type II error issue that was caused by floating point round-off, the INL proposed to develop an integer based algorithm for time-stepping to replace it. The fundamental reason was that integer addition, subtraction and multiplication are exact; there is no round-off. However, as PVMEXEC and other all codes connecting with RELAP5-3D use real time for output, communication and timecard end, the integer algorithm must also use floating point values for these.

Use of integers to count time is not a new idea. Though time is inherently a continuous quantity that is best represented by floating point values on a computer, integer-based time is used in all computers. Computer chip speed is rated in terms of the number of billion operations it can 


\section{Implementation of a New DTSTEP Algorithm for use in RELAP5-3D and PVMEXEC}

\section{Completion Report}

perform in a second in $\mathrm{GHz}$ or clock-cycles. A clock-cycle is the amount of time to perform the simplest operation or one tick of its clock. The integer basis for time stepping is also the clockcycle or tick and that is defined to be the smallest timestep that RELAP5-3D can take and is denoted $\Delta \mathrm{t}_{\text {small. }}$. It is generated by the user's selection of $\Delta \mathrm{T}_{\max }$ and $\Delta \mathrm{T}_{\min }$. An example is shown in Figure 2 with real time denoted $\mathrm{t}$, integer time denoted $\tau, \Delta \mathrm{t}_{\max }=0.004$, and $\Delta \mathrm{t}_{\min }=1.0 \mathrm{e}-7$.

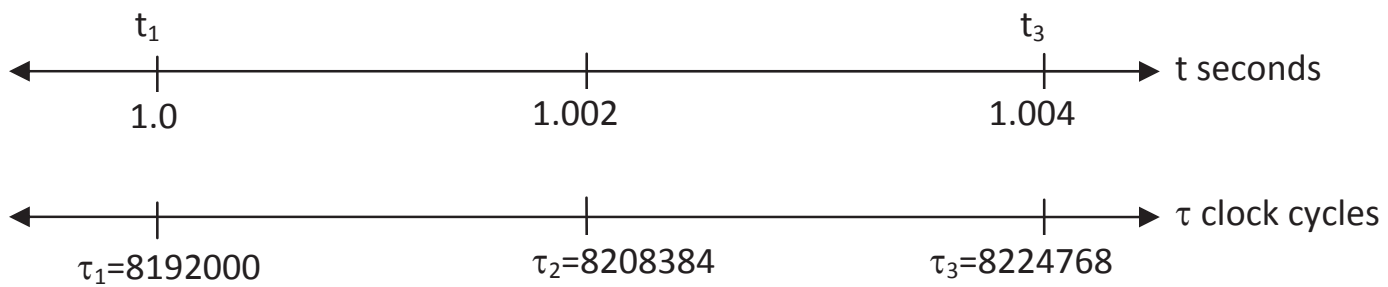

Figure 2. Correspondence between integer and real time

In this case, the integer clock cycle value $\tau=1$ tick, represents real time $\Delta \mathrm{t}_{\text {small }}=1.220703125 \times 10^{-7}$ and $\Delta \tau_{\max }=2^{15}$ because $2^{15} \times\left(1.22 \times 10^{-7}\right)=0.004$. Thus, $\mathrm{t}_{1}=1.000$ corresponds to $\tau_{1}=250 \times 2^{15}=$ 8192000 , and $\tau_{2}=\tau_{1}+2^{14}=8208384$ because $.002=2^{14} \times\left(1.22 \times 10^{-7}\right)$.

The formulae that generate the correspondence between reals and integers are given New Algorithm 1 which encompasses Eq. (6), (6a), and (6b).

New Algorithm 1. Integer/Real Time Initialization (start of transient)

1. $H=$ Floor $\left[\log _{2}\left(\frac{\Delta \mathrm{T}_{\max , C}}{\Delta \mathrm{T}_{\min , C}}\right)\right]=$ Floor $\left[\log \left(\frac{\Delta \mathrm{T}_{\max }}{\Delta \mathrm{T}_{\min }}\right) / \log (2)\right]$

2. $\Delta \tau_{\max }=2^{H}$

3. $\Delta \mathrm{t}_{\text {small }}=\Delta \mathrm{T}_{\max } / \Delta \tau_{\max }=\Delta \mathrm{T}_{\max } / 2^{\mathrm{H}}$

Note: the floor function in Eq. (6) truncates real numbers by removing the fractional portion. In Figure $2, \log (.004 / .0000001) / \log (2) \approx 15.28$; so $\mathrm{H}=$ Floor $[15.28]=15, \Delta \tau_{\max }=2^{15}=32768$, and $\Delta \mathrm{t}_{\text {small }}=.004 / 32768=0.0000001220703125$.

There is no concern about restricting the range of floating point values that can be represented by 64-bit integers. A 64-bit integer can represent values in excess of $9 \times 10^{18}$. This is sufficient, for example, to exactly calculate every integer multiple of $\Delta \mathrm{t}_{\text {small, }}$, between $\Delta \mathrm{T}_{\mathrm{MIN}}=10^{-9}$ and $\mathrm{T}=10^{9}$ seconds.

Integer timestepping has replaced real timestepping in RELAP5-3D and PVMEXEC. However, most of the complication occurs in RELAP5-3D; therefore most of the ensuing discussion is presented from the perspective of the RELAP5-3D code. Reference is made to PVMEXEC where helpful and in the PVMEXEC section. 


\section{Implementation of a New DTSTEP Algorithm for use in RELAP5-3D and PVMEXEC}

Completion Report

\subsection{Integer Timestep Basics}

This $\Delta t_{\text {small }}$ is the smallest value of $\Delta t$ that satisfies rules (1) and (2), but it can change on every timecard, $\mathrm{C}$, because the user can change the max and min timestep, $\Delta \mathrm{T}_{\max , \mathrm{c}}$ and $\Delta \mathrm{T}_{\min , \mathrm{c}}$. The calculations for crossing to timecard C-1 to timecard C are given in New Algorithm 2.

\section{New Algorithm 2. Timecard crossing}

1. $H(C)=F \operatorname{loor}\left[\log \left(\frac{\Delta \mathrm{T}_{\max , C}}{\Delta \mathrm{T}_{\min , C}}\right) / \log (2)\right]$

2. $\Delta \tau_{\max , \mathrm{C}}=2^{H(C)}$

3. $\Delta \mathrm{t}_{\text {small, } \mathrm{C}}=\Delta \mathrm{T}_{\max , \mathrm{c}} / \Delta \tau_{\max , \mathrm{C}}$

4. $\tau_{\mathrm{C}}=0$

Step 4 is the only real difference from New Algorithm 1. It restarts integer cumulative time at zero with every timecard while floating-point cumulative time is unaffected. Throughout the time controlled by timecard $\mathrm{C}$, integer cumulative time, floating point $\Delta \mathrm{t}$, and floating point cumulative time are calculated by the Equations of New Algorithm 3. Note that $\mathrm{T}_{\mathrm{C}-1}$ is the endtime of the timecard C-1; it is also the beginning time of timecard C.

\section{New Algorithm 3. Integer/Real time advancement (for chosen $\Delta \tau_{i}$ )}

1. $\tau_{c, i}=\tau_{c, i}+\Delta \tau_{i}$

2. $\Delta \mathrm{t}_{\mathrm{i}}=\Delta \mathrm{t}_{\mathrm{small}, \mathrm{c}}{ }^{*} \Delta \tau_{\mathrm{i}}$

3. $\mathrm{T}_{\mathrm{i}}=\mathrm{T}_{\mathrm{C}-1}+\tau_{\mathrm{c}, \mathrm{i}}^{*} \Delta \mathrm{t}_{\mathrm{small}}$

Floating point cumulative time and time targets sent at a communication points by PVMEXEC to RELAP5-3D must be converted to integers. Time targets are communication, output, or timecard end times. Floating point time targets may not be representable exactly by an integer multiple

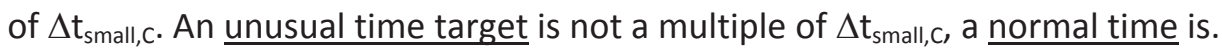

An unusual time can be created by user selection of a timecard endtime that is not a multiple of $\Delta T_{\max }$. It can also be caused by PVMEXEC having a different $\Delta \mathrm{T}_{\max }$ than RELAP5-3D. Time targets,

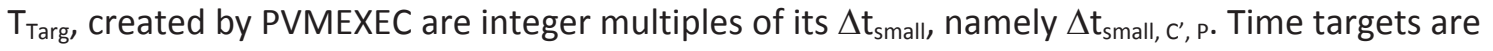
unusual if they are not multiples of the $\Delta t_{\text {small }}$ of RELAP5-3D, namely $\Delta t_{\text {small, }, R \text {. }}$.

If $\underline{\underline{T}}_{\text {Targ }}$ is unusual, there is no exact integer representation for it. The corresponding value on the $\tau$-timeline lies between two consecutive integers. Figure $2 \mathrm{a}$ has $\mathrm{T}_{\mathrm{Targ}}=1.0021$ and $\mathrm{T}_{\mathrm{C}-1}=1.0$; thus $\tau_{\text {left }}=\left[\left(T_{\text {Targ }}-T_{c-1}\right) / \Delta t_{\text {small }}\right]=8192000+17203$ (truncated) and $\tau_{\text {right }}=\tau_{\text {left }}+1=8209204$.

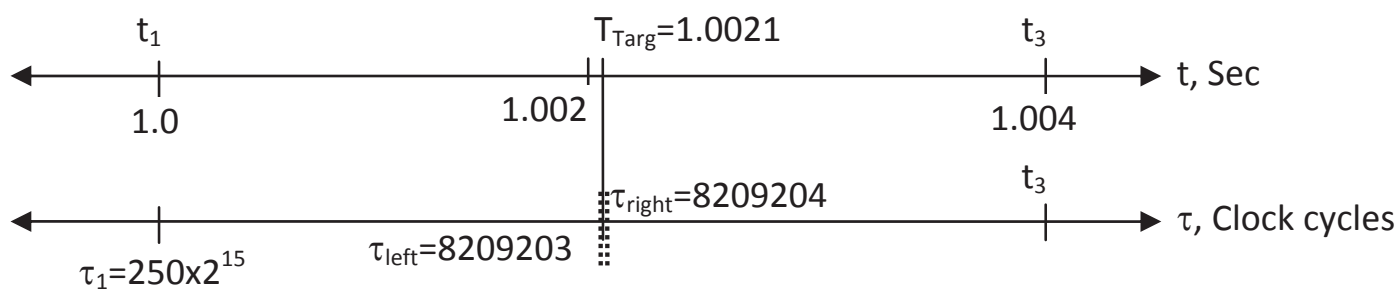

Figure 2a. Unusual times cannot be represented exactly by an integer. 


\section{Implementation of a New DTSTEP Algorithm for use in RELAP5-3D and PVMEXEC}

Of course, if $\underline{T}_{T a r g}$ is a normal time, then integer $\tau_{\text {Targ }}=$ Round $\left[\left(T-T_{C-1}\right) / \Delta t_{\text {small }}\right]$ exactly represents the real time target and there are no issues reaching it.

Let $T_{E, k}$ be the next minor edit, plot, major edit, restart, and PVM-communication points from the PVM message, respectively, and let $\tau_{E, k}$ be the integer equivalents. The conversion of floating point time quantities to integers is given in New Algorithm 4. Rounding is necessary to prevent automatic truncation of fractional portions of real quotients in integer conversion. For example, applying Eq. (8) to Table 3 with $\mathrm{T}=1.0009990842492, \Delta \mathrm{t}_{\text {small }}=1.52587887214593 \times 10^{-7}$ and $\mathrm{T}_{\mathrm{C}}=$ 1.0 , yields $\tau_{\mathrm{C}}=6547$ without rounding, but 6548 with rounding.

New Algorithm $4 . \quad$ Convert real time quantities to integers

1. $\tau_{\mathrm{C}}=\mathrm{f}\left(\mathrm{T}, \mathrm{T}_{\mathrm{C}-1}, \Delta \mathrm{t}_{\text {small }}\right) \equiv \operatorname{Round}\left[\left(\mathrm{T}-\mathrm{T}_{\mathrm{C}-1}\right) / \Delta \mathrm{t}_{\text {small }}\right]$

2. $\Delta \tau_{\mathrm{i}}=\operatorname{Round}\left[\Delta \mathrm{t}_{\mathrm{i}} / \Delta \mathrm{t}_{\text {small }}\right]$

3. $\tau_{E, k}=\operatorname{Round}\left[\left(T_{E, k}-T_{C-1}\right) / \Delta t_{\text {small }}\right], j=1, \ldots, 5$

This algorithm applies to unusual or normal time quantities. However for unusual quantities, the integer does not exactly represent the floating point quantity.

Another important concept is the so-called perfection of the timestep, $\Delta t$. This means making a normal timestep into an exact multiple of $\Delta \tau_{\text {small }}$. It is necessary since floating point calculations of $\Delta t$ elsewhere in the overall DTSTEP algorithm may result in a $\Delta t_{i}$ that is not an integer multiple of $\Delta \tau_{\text {small. }}$ Just before advancing time, a normal timestep is perfected with New Algorithm 5.

\section{New Algorithm 5. Perfect a normal timestep}

1. Normal $=\left(\left|\tau_{i+1} * \Delta t_{\text {small }}-\Delta t_{i+1}\right|<\delta\right)$

If (Normal) then
a. $\Delta \mathrm{t}_{\mathrm{i}+1}=\Delta \mathrm{t}_{\text {small, }, \mathrm{c}} \mathrm{x} \Delta \tau_{\mathrm{i}+1}$
b. $T_{i}=T_{C-1}+\left(\tau_{c, i}\right) x\left(\Delta t_{\text {small }}\right)$

(7a repeated)

( $7 \mathrm{~b}$ repeated)

If the timestep is close to a multiple of $\Delta \tau_{\text {small, }}$ then inexactness in its last bits resulting from roundoff in its calculation is eliminated by (7a). The same is done for cumulative time. Since $\Delta \mathrm{t}_{\mathrm{i}+1}$ does not arise from a sum of many terms, accumulation of roundoff is not an issue; thus roundoff is confined to the last one or two bits, depending upon the calculation. Therefore, $\delta$ is taken to be a very tight tolerance. Initially, $\delta=10^{-10}$ was used; however, for very large timesteps, such as in the ans79.i input model with timesteps on the order of $10^{6}$, the tolerance must scale with the size of the timestep, and therefore $\delta=\Delta \mathrm{t}_{\mathrm{i}+1} \times 10^{-10}$ was used.

These conversions provide a basis for replacement of floating point tolerance tests with integer equality tests for reaching a time target. This issue is explained in Section 4.1.

\subsection{Integer Time Equality Tests}

If $\underline{I}_{\text {Targ }}$ is normal, then integer $\tau_{\text {Targ, }}$, defined via Eq. (8b), exactly represents the real time target and there are no issues reaching it. For unusual time targets there is considerable complication. 


\section{Implementation of a New DTSTEP Algorithm for use in RELAP5-3D and PVMEXEC}

\section{Completion Report}

The idea for handling unusual times is to consider two linked timesteps that will reach from the current advancement, $i$, at time $T_{i}$ to advancement $i+2$ at $T_{i+2}=T_{i}+2 \Delta t_{i}$. During the first linked step, integer and real time do not correspond exactly. The timestep used by RELAP5-3D during advancement $i+1$ is the value of $\Delta t$ that exactly reaches $T_{\text {TARG }}$. The second step reaches $T_{i+2}$.

\section{New Algorithm 6: $\quad$ Unusual Time Target Handler}

If (UH is false) $U H=\left(T_{i}+\Delta t_{i+1} \geq T_{\text {TARG }}\right)$ and ( $T_{\text {TARG }}$ is unusual)

Linked Advancement 1 when $\mathrm{UH}$ is true

1. $\Delta \mathrm{t}_{\mathrm{i}+1}=\mathrm{T}_{\mathrm{TARG}}-\mathrm{T}_{\mathrm{i}}$

2. $\Delta \tau_{\mathrm{i}+1}=\tau_{\text {left }}-\tau_{\mathrm{i}}$

3. $\tau_{\text {save }}=\tau_{\mathrm{i}}+2 \Delta \tau_{\mathrm{i}}$

4. $T_{\text {save }}=T_{i}+2 \Delta t_{i}$

Linked Advancement 2, when UH is true,

1. $\Delta \mathrm{t}_{\mathrm{i}+2}=\mathrm{T}_{\text {save }}-\mathrm{T}_{\mathrm{TARG}}$

2. $\Delta \tau_{i+2}=\tau_{\text {save }}-\tau_{i+1}$

3. $\mathrm{UH}=$ false

Variable UH indicates that the unusual handler is active when true, otherwise it is false. Once the handler is on, UH keeps it on until the second linked advancement is finished. There are two complications with New Algorithm 6. The first is determining that $\mathrm{T}_{\text {TARG }}$ is unusual; see Section 4.1.1. The second occurs when the second linked step is halved by other portions of the DTSTEP algorithm; see 4.1.2. Also, this algorithm does not cover the $110 \%$ stretch logic of rule (4).

\subsubsection{Normal / Unusual Testing}

Algorithm 6 applies to unusual targets, those that are not exactly a multiple of $\Delta t_{\text {small }}$. However, it is important that times very close to multiples of $\Delta t_{\text {small }}$ be considered normal also. Otherwise accumulation of any roundoff error would cause virtually every time value produced via floating point summing to test as unusual. Another consideration besides roundoff is the variation in the quotients when divided by $\Delta \mathrm{t}_{\text {small. }}$. Quotients of larger numbers, such as $\mathrm{T}$, can have less accuracy than those of smaller numbers, such as $\Delta t$. Therefore, determination of unusual time target is better served by basing the test for normality on $\Delta t$ rather than $T_{\text {TARG }}$.

There are two coupling modes based on control of the timestep by PVMEXEC. In asynchronous coupling modes, all codes proceed to a communication point with their own selection of $\Delta t$ at every step. In synchronous mode, PVMEXEC supplies $\Delta \mathrm{t}_{i+1}$ to all coupled codes at every step, and all codes must use that timestep. This is summarized in Eq. (9).

$$
\Delta t_{i+1}=\left\{\begin{array}{l}
T_{T A R G}-T_{i} \quad \text { if asynchronous } \\
\Delta t_{P V M E X E C, i+1} \text { if synchronous }
\end{array}\right\}
$$

With the timestep selected, the determination of unusualness is made with New Algorithm 7. 


\section{Implementation of a New DTSTEP Algorithm for use in RELAP5-3D and PVMEXEC}

Completion Report

New Algorithm 7.

Time Target Unusualness Test

1. $\Delta \tau_{\text {check }}=\operatorname{Round}\left[\Delta \mathrm{t}_{\mathrm{i}+1} / \Delta \mathrm{t}_{\text {small }}\right]$

2. $\Delta \mathrm{t}_{\text {ch }}=\Delta \tau_{\text {check }} * \Delta \mathrm{t}_{\text {small }}$

3. UNUSUAL $=\left|\Delta \mathrm{t}_{\mathrm{ch}}-\Delta \mathrm{t}_{\mathrm{i}+1}\right|>0.01 \times \Delta \mathrm{t}_{\text {small }}$

Algorithm 7 sets UNUSUAL to true when the target is an unusual time. Since $\Delta \mathrm{t}_{\mathrm{i}+1}$ and $\Delta \mathrm{t}_{\mathrm{ch}}$ have the same size and agree for many leading digits. The number of leading digits that must agree determines unusualness. Requiring too many allows accumulation of roundoff to falsely indicate unusual status when the target is normal. Not requiring enough allows some unusual targets to falsely test as normal. This is a Type I vs. Type II error situation.

Note that for smaller timesteps than $\Delta t_{\max }$, the subtraction in Eq. (9) reduces the number of significant digits available to compare. The test reflects this loss. Comparison against $0.01 \times \Delta t_{\text {small }}$ in Eq. (10b) requires at least two decimal places of agreement when $\Delta t_{i+1}$ is close to $\Delta t_{\text {small }}$ in size, and requires more places of agreement for larger $\Delta \mathrm{t}_{\mathrm{i}+1}$. Typically, $\Delta \mathrm{T}_{\max } / \Delta \mathrm{T}_{\min } \approx 10^{6}$, so typically, agreement to $6+2=8$ places is required for $\Delta \mathrm{t}_{i+1}$ near $\Delta \mathrm{T}_{\max }$. For IEEE 64-bit floating pt. representation, approximately 15 digits can be represented fully. Therefore, agreement to the first 8 places ignores the lowest $7=15-8$ respectively.

Since New Algorithm 5 perfects every normal timestep and its associated cumulative time, $T_{i}$, only the final bit of $T_{i}$ is subject to roundoff error. However for a large cumulative time, the difference $\Delta \mathrm{t}_{i+1}=\mathrm{T}_{\text {Targ }}-\mathrm{T}_{\mathrm{i}}$ in Equation (9) can account for a substantial loss of significant digits, creating an inaccuracy in the lowest 7 decimal places. This problem is itself mitigated on most modern platforms that use 96 bits for floating point operations (effectively adding 10 extra digits to the calculation) before rounding to store the value. Thus it is very difficult, but possible if all 15 digits of time are important, for New Algorithm 7 to fail.

It is possible to improve New Algorithm 7 so that failure is even less likely. By combining Eq. (6b), (2) and (7a) it is seen that a normal integer timestep is always a power of two.

$$
\begin{aligned}
& \Delta \mathrm{t}_{\text {small }}=\Delta \mathrm{T}_{\text {max }} / 2^{\mathrm{H}} \text {, so that } \quad \Delta \mathrm{T}_{\text {max }}=2^{\mathrm{H}} \Delta \mathrm{t}_{\text {small }} \\
& \Delta \mathrm{t}_{\mathrm{i}}=\Delta \mathrm{T}_{\text {max }} / 2^{\mathrm{k}}=\Delta \mathrm{t}_{\text {small }} 2^{\mathrm{H}} / 2^{\mathrm{k}}=\Delta \mathrm{t}_{\text {small }} 2^{\mathrm{H}-\mathrm{k}} \\
& \Delta \tau_{\mathrm{i}}=\Delta \mathrm{t}_{\mathrm{i}} / \Delta \mathrm{t}_{\text {small }}=2^{\mathrm{H}-\mathrm{k}} .
\end{aligned}
$$

Any timestep taken to reach a time target whose integer representative is not a power of two is automatically unusual. A simple test for this is given in New Algorithm 8.

\section{New Algorithm 8. Power of two test for normality}

1. $H=\operatorname{Round}\left[\log \left(\Delta \tau_{i+1}\right) / \log (2)\right]$

2. $N O R M A L=\left(\Delta \tau_{i+1}\right.$.eq. $\left.2^{H}\right)$

The combination of Algorithms 7 and 8 provides a better test for normality or unusualness than Algorithm 7 alone. 


\section{Implementation of a New DTSTEP Algorithm for use in RELAP5-3D and PVMEXEC}

\subsubsection{The Unusual Handler's second linked step}

The second linked step of Algorithm 6, Unusual Time Target Handler, must handle two cases:

(1) $\Delta t$ is halved by other portions of the DTSTEP algorithm or

(2) The advancement is repeated.

Case (2), repeating either the first or second advancement causes a special logical variable to be set to indicate that time backed up by one timestep. On the next pass through DTSTEP, the unusual handler is cancelled.

Case (1), if the first linked step succeeds and the second is halved and repeated, the saved target $\mathrm{T}_{\mathrm{i}+2}$ cannot be reached on the second step. However, the handler is cancelled by the backup and the rest of the DTSTEP algorithm will create an appropriate timestep.

\subsection{Integer equality tests}

With these definitions, conversion equations, and algorithms in place, all the floating point tolerance tests were replaced with integer equality tests.

Replace statements with this form,

$$
\text { If }\left(\mathrm{abs}\left(\mathrm{T}_{\mathrm{i}}+\Delta \mathrm{t}_{\mathrm{i}}-\mathrm{T}_{\mathrm{TARG}}\right)<\varepsilon\right) \text { action }
$$

With

$$
\text { If }\left(\tau_{i}+\Delta \tau_{i} \text {.eq. } \tau_{\text {TARG }}\right) \text { action }
$$

There were also tests that required integer inequalities, such as stretch logic. There were a couple of tests that had to remain floating point, such as a Courant limit test. Converting all tests proved insufficient to solve all the problems with the PVM coupling of RELAP5-3D to other codes. Some of these are covered in Section 5.

\subsection{Integer Time Advanced Concepts}

Advanced concepts covers the solution to difficult programming issues due either to complexity in the algorithm underlying the original coding, or to the need to create alternative means to implement with integers an algorithm designed for floating point calculations. The latter were often revealed by runtime errors. Most errors with the integer time step involved integer cumulative time differing from a time target by exactly one. Most of these involved time targets generated by PVMEXEC and sent to RELAP5-3D. Under close examination, the source of each of these was tracked and solutions devised.

Before examining these issues, it is important to note two conditions that were placed upon the workscope as they restricted the choice of solutions. The development and implementation of the integer algorithm was carried out under two constraints:

1. The functionality of DTSTEP must remain the same.

2. The PVM messages cannot be altered in any way, neither by adding new communication messages nor by modifying any existing message. 


\title{
Implementation of a New DTSTEP Algorithm for use in RELAP5-3D and PVMEXEC
}

\author{
Completion Report
}

The first constraint was put in place to ensure that the tens of thousands of existing input models would continue operating exactly the same before and after the change to the algorithm, except that problems which failed under the tolerance test were to run under the integer algorithm. It was recognized cumulative time would be slightly different because of more accurate temporal calculation; however this is not a change of functionality.

The second constraint was put in place to preclude the necessity of modifying all the computer codes that had previously been programmed to communicate with RELAP5-3D and PVMEXEC. Although efficient at reducing overall rework, two exceptions to this constraint proved necessary for solving difficult issues; these were eventually implemented.

In the interest of brevity, only five of the many and complex advanced issues are covered, and only from a high level perspective. Those addressed include: handling minimal timesteps in Sec. 5.1, finding a target halving of $\Delta \mathrm{T}_{\max }$ in Sec 5.2, timecard crossing in Sec 5.3, message exchange time in Sec 5.4, the 110\% stretch logic in Sec 5.5, and the simplification of the synchronous coupling handler in Sec. 5.6.

\subsection{Handling Minimal Timestep}

The DTSTEP algorithm originally automatically halved a timestep if certain conditions occurred, such as excessive mass error, even if already minimal, temporarily making $\Delta \mathrm{t}_{i+1}=\Delta \mathrm{t}_{\text {small }} / 2$. Later, the algorithm would correct this by doubling $\Delta \mathrm{t}_{i+1}$ if below $\Delta \mathrm{T}_{\text {min }}$. The reduction below $\Delta \mathrm{T}_{\text {min }}$ occurred in several places in DTSTEP. This algorithm does not work for integers because the smallest integer is 1 ; halving 1 produces 0 , and doubling 0 produces 0 , not 1 .

The solution was to rework the DTSTEP algorithm so that timesteps were not allowed, under any circumstances, to go below 1 for integers and for $\Delta T_{\min }$ for reals, and automatic doubling was adjusted to account for this. In compliance with the requirement that DTSTEP functionality be unchanged, it is noted that this had no effect on the functionality of DTSTEP. See Section 5.0.

\subsection{Exceptions to the halving and doubling logic for $\Delta T_{\max }$}

There are several instances in the overall DTSTEP algorithm when the current $\Delta t$ does not fit the DTSTEP halving and doubling scheme, rule (2). These instances are:

1. When crossing to a timecard with a different $\Delta \mathrm{T}_{\max }$, different $\Delta \mathrm{T}_{\min }$, or both

2. When $\Delta T$ is calculated to satisfy Courant limit

3. After a $110 \%$ stretch to a time target has occurred

4. After a compression to take 2 equal steps to reach a time target

In all these cases, the time step in use does not match the form of rule (2). Return to the correct form is required so that the halving/doubling logic of DTSTEP works properly. This is done in accordance with Section 5.0 requirement that the functionality of DTSTEP not be changed.

In the original DTSTEP algorithm, a timestep halving loop starting at the controlling value of $\Delta t_{\max }$ and halving the candidate timestep was used. It stopped with the first value less than or equal to the target value. It could produce a value below $\Delta \mathrm{t}_{\text {small }}$. 


\section{Implementation of a New DTSTEP Algorithm for use in RELAP5-3D and PVMEXEC}

This is unworkable for the integer algorithm as explained in Section 5.1. New Algorithm 9 was created that stopped if $\Delta \mathrm{t}_{\text {candidate }}<\Delta \mathrm{t}_{\text {small, }}$ and set $\Delta \mathrm{t}_{\text {candidate }}=\Delta \mathrm{t}_{\text {small }}$ and set $\Delta \tau_{\text {candidate }}$ using Eq. (8a). If the target was negative, it set the failure flag to true so that the RELAP5-3D calculation could terminate gracefully.

\section{New Algorithm 9. Find Halving $\leq$ target}

If (target < 0.0) then

$$
\text { Fail }=\text { true }
$$

Else if (target $<\Delta \mathrm{t}_{\text {small }}$ ) then

$$
\Delta \mathrm{t}=\Delta \mathrm{t}_{\text {small }}
$$

Else

Loop (on $\mathrm{k}$ ) to find $\Delta \mathrm{t}=(0.5)^{\mathrm{k}} \Delta \mathrm{T}_{\mathrm{BASE}} \leq$ target

where $\Delta \mathrm{T}_{\text {BASE }}$ is either $\Delta \mathrm{T}_{\mathrm{MAX}}$ or an unusual time-step

Endif

$\Delta \tau=\operatorname{Round}\left[\Delta \mathrm{t} / \Delta \mathrm{t}_{\text {small }}\right]$

Note that the halving is actually performed by multiplication by the reduction factor of 0.5 . The test for negative target is included because during the debugging, negative timesteps were created in other portions of DTSTEP and this was introduced to catch those and stop gracefully.

\subsection{Timecard crossing and endtime}

From the original DTSTEP algorithm, a timecard crossing is governed by the logical variable LAST. This variable was set differently for PVM synchronous control, for which it could only be true at the transient end, than for all other modes of operation where it is true at timecard endtimes.

Under PVM asynchronous control or autonomous RELAP5-3D control, LAST became true only when, before a timestep can be taken, cumulative time is already past the timecard endtime. The integer equivalent test was an inequality, rather than an equality such as Eq. (11).

Under synchronous control, this test was restricted to transient endtime as calculated from the values of $\Delta T_{\max }$ and $\Delta T_{\min }$ and $T_{\text {end }}$ on the most recent PVM communication of these values. Synchronous control was maintained according to Requirement 2 of Sec 5.0.

\section{$5.4110 \%$ stretch logic}

The stretch logic was applied to reaching every unusual time target. For PVM coupling, DTSTEP included stretch logic for plot, minor edit, major edit, restart, communication time and timecard endtime. There were two issues to resolve, conversion to integers and the proper order.

This test required a conversion to integers for the testing, but use of floating point time to calculate the potentially unusual timestep. In some cases, the $110 \%$ stretch logic actually caused a timestep to be shortened to an unusual step to exactly reach the next target time. Finally, the algorithm for processing the stretch differed between autonomous control, PVM synchronous and PVM explicit time control in RELAP5-3D. 


\title{
Implementation of a New DTSTEP Algorithm for use in RELAP5-3D and PVMEXEC
}

\author{
Completion Report
}

With explicit time control in use, the explicit message exchange time was the most important time to reach. It was necessary to override the $110 \%$ stretch when taking a step of that size would step past an explicit message exchange time to reach a larger time target; that would result in a "code hang" or "hung machine." Both mean that one code is waiting for one kind of message while the other code waited for another, and no message is being sent by either. This created in an interminable period of waiting, otherwise called a "code hang," and required the coupled calculation to be terminated by outside intervention.

This induced a requirement to check and insure that explicit exchange time could never be bypassed. The functionality was preserved according to requirement 2 of Sec 5.0, but the algorithm was adjusted to account for this and solutions to issues identified during debugging. Subroutine dthyCalc (see Table $7 \mathrm{~b}$ ) embodies this algorithm; so it is not given here.

\subsection{Message and Target Time}

The time to exchange messages was a major source of programming issues for PVM coupling between RELAP5-3D DTSTEP and other codes, including PVMEXEC DTSTEP. Roundoff error often caused the codes to meet the exchange time condition on a different timestep resulting in a hung machine. This was an issue for both synchronous and asynchronous coupling.

Depending on whether coupling is synchronous, asynchronous, or standalone, different logic paths and calculations are performed and the messages are different or non-existent. The programming was complicated by insufficient data for the original RELAP5-3D DTSTEP algorithm that required end of timecard. No successful substitute for that datum was found; so eventually it had to be added despite Section 5.0 rule 2 . Integer calculations were further complicated by exchange times that were unusual times.

To reduce rounding errors when converting floating point times from messages to integers, message times were recalculated, based on the controlling $\Delta T_{\max }$ and $\Delta T_{\min }$ at every message exchange. In fact for the same reason, whenever one target time was reached, every target time was recalculated.

\subsection{Synchronous Mode Simplification}

In the original implementation of the integer-based timestep size selection and advancement algorithm, there were situations in which RELAP5-3D, operating in a synchronous mode where the ultimate timestep size is determined by PVMEXEC, was performing calculations of the timestep size needed to reach special communication intervals. The logic that was used for some of the calculations was inconsistent between RELAP5-3D and PVMEXEC, but more importantly, the logic was completely superfluous. However, according to requirement 2 of Section 5.0, this superfluous logic and coding could not be eliminated.

After numerous efforts to correct the inconsistencies between RELAP5-3D and PVMEXEC, it was decided to make another exception to Sec. 5.0 requirement 2 . This resulted in essentially a new algorithm for handling synchronous coupling. In the new algorithm, RELAP5-3D reports a timestep size back to PVMEXEC that depends only on the built-in error-checking and errormitigation processes in the code, including the prohibition of violating the Courant Limit for 


\title{
Implementation of a New DTSTEP Algorithm for use in RELAP5-3D and PVMEXEC
}

\author{
Completion Report
}

RELAP5-3D problems that use the semi-implicit time advancement scheme. This represents a significant reduction in the logic associated with time step size selection for synchronously coupled calculations.

\subsection{Integer Time Implementation}

Initial status is in Sec 6.1. The implementation had many parts: mapping DTSTEP functionality in Sec 6.2; moving code into collections according to functionality in Sec 6.3; defining, eliminating \& mnemonically renaming variables in Sec 6.4; creating a module for integer data in Sec 6.5; and creating internal subroutines in Sec 6.6.

\subsection{Initial Status}

The original status of RELAP5-3D DTSTEP was poor. There was little documentation, numerous GOTO statements (both forward and backward) which made tracing the logic flow extremely difficult, non-mnemonic variable names, large amounts of inert or unused code marked with pre-compiler directives, even some sections of dead code. Over a dozen authors had written code and modified it; thus many programming styles were present. All this made the main algorithm hard to read and understand.

The PVMEXEC DTSTEP subroutine was created from the RELAP5-3D DTSTEP subroutine by removing coding unneeded for controlling the coupled calculation and then adding the communication and decision logic to operate as the executive. It had many of the same issues.

There were a number of existing and unresolved user problems before this project began (all of which have since been resolved); Table 1 lists several and indicates that debugging these two subroutines had been ongoing for 5 years. Moreover, there were also a number of undiscovered errors that this project revealed and resolved. Together, the pair of subroutines had algorithmic and coding issues as well as a number of errors.

\subsection{DTSTEP Functionality}

Mapping the DTSTEP functionality was the necessary starting point. It supported requirement one of Section 5.0 and proved essential to all ensuing work. The following is a high-level summary of the functionality within RELAP5-3D DTSTEP after removing all coding that is either inert or otherwise unused.

\section{A. AUTOMATIC FUNCTIONS}

1. Initialization

- Unconditional initialization

- Initialization carried out on the first transient step only

- Card-one initialization

2. Advancement statistics collection

B. END OF ATTEMPTED-ADVANCEMENT PROCESSING

3. Time step success evaluation

- Based on RELAP5-3D criteria, set indicator of step success, failure or backup

- If synchronous hydraulic coupling, (A) send indicator to PVM executive, (B) Receive global success flag from PVM executive 


\title{
Implementation of a New DTSTEP Algorithm for use in RELAP5-3D and PVMEXEC
}

\author{
Completion Report
}

- For failure - restore old time step, time and data, activate debug

- For backup - reduce time step and time, restore old data

4. Transient/steady-state termination conditions

- RELAP5-3D termination conditions

- Normal - Transient terminated by end of time step cards

- Transient terminated by trip

- Transient terminated by approach to CPU time limit.

- Transient has reached steady state.

- Transient terminated by interactive command.

- Transient terminated by Card 105 input option.

- Transient terminated by variable volume model.

- Transient terminated by failure in PVM coupled computation

- Help operations

5. Time targeting

- *Current \& next edit time- Based on time-step card and PVM target time data

- *Exact arrival at time target - New integer-based time-target algorithm

\section{SET-UP / PREPARATION FOR "NEXT" ATTEMPTED-ADVANCEMENT}

6. PVM communication

NOTE: this is only done if success is indicated and it is not the last advancement.

- If at explicit asynchronous hydraulic coupling communication time, send/receive appropriate messages

- If explicit synchronous hydraulic coupling, send/receive appropriate messages

- If at a PVM (edit or explicit coupling time) target time, receive new set of PVM target times

7. RELAP5-3D time step calculation

Note: only the final selection sub-function is performed if success flag indicates failure.

- *Time step card control processing -control from current card, next card?

- *Courant limit calculation(s)

- *Time step halving and doubling - RELAP5-3D conditions that change time-step size

- Hitting the target - stretch above max, shrink below min, two equal steps

- Final selection of time step - Based on success/failure control, normal step selection, and hitting target. Update cumulative time and advancement count.

8. PVM synchronous time step communication

- Send synchronous message - Transmit time-step to PVM executive

- Receive synchronous message - Get global time-step

D. OUTPUT

9. Code output

- Screen text

- File output

- Printed output file (major and minor edits)

- Restart-plot files (plot records and restart records, sequential \& direct access)

- Dump files (binary files controlled by 105-card input)

- Additional debug output (controlled by failure modes, 105-card input) 


\section{Implementation of a New DTSTEP Algorithm for use in RELAP5-3D and PVMEXEC}

\section{Completion Report}

Note that the four major sections and nine subsections are arbitrary and introduced by the author merely to enhance understanding. The coding showed no such breakouts, nor did the coding for all these sections exist in contiguous blocks.

\subsection{DTSTEP Reorganization}

These functions identified in Sec 6.2 were reorganized into 11 major sections and coding was moved to collect the statements that worked together to perform these functions. This was done without affecting the logic flow. Figures $3 a$ and 3 b give a "sectional flowchart" of the reorganization of DTSTEP. 


\section{Implementation of a New DTSTEP Algorithm for use in RELAP5-3D and PVMEXEC}

\section{Completion Report}

\begin{tabular}{|c|c|}
\hline $\begin{array}{l}\text { 1. Initialize } \\
1 \text { Initial logic flow control flags } \\
2 \text { Initialize time step control variables } \\
3 \text { if (.not.skipt) "First entry only initializations" } \\
4 \text { set nmechk, CPU-time }\end{array}$ & \multirow[t]{24}{*}{\begin{tabular}{l}
\multicolumn{1}{c}{ NOTE } \\
This sectional flow \\
diagram shows no \\
arrows for jumps \\
between or within \\
sections.
\end{tabular}} \\
\hline 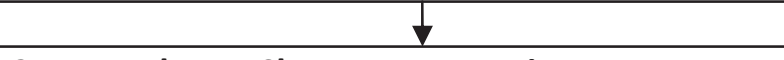 & \\
\hline $\begin{array}{l}\text { 2. Abrupt Changes to Transient } \\
\text { (Terminate, halve/double, repeat) } \\
1 \text { Evaluate various immediate terminal tests } \\
2 \text { Handle failed advancements and terminations } \\
3 \text { Handle halve/double, minimum step, repeating }\end{array}$ & \\
\hline 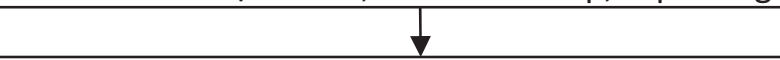 & \\
\hline $\begin{array}{l}\text { 3. Gather statistics } \\
\text { (Done for major edits) }\end{array}$ & \\
\hline$\downarrow$ & \\
\hline $\begin{array}{l}\text { 4. New Advancement } \\
1 \text { Conditional advancement to next timecard } \\
2 \text { The advancements are to continue. } \\
3 \text { Test for steady state, subroutine sstchk }\end{array}$ & \\
\hline 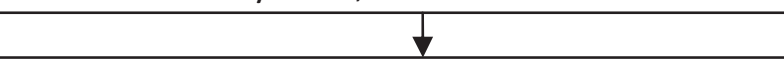 & \\
\hline Code Output & \\
\hline Screen output & \\
\hline Plots on disk for internal plots & \\
\hline Minor edits & \\
\hline Restart records on restart-plot file & \\
\hline R5-Force & \\
\hline Plot records on restart-plot file & \\
\hline Nuclear Plant Analyzer & \\
\hline Compression: sqoz, comprs, snaptrans & \\
\hline Direct-access restart plot file & \\
\hline$\downarrow$ & \\
\hline PVM 8002-8005 message exchanges & \\
\hline Explicit parallel synchronous (8002) & \\
\hline Explicit parallel asynchronous (8003) & \\
\hline Explicit sequential synchronous (8004) & \\
\hline Explicit sequential asynchronous (8005) & \\
\hline
\end{tabular}




\section{Implementation of a New DTSTEP Algorithm for use in RELAP5-3D and PVMEXEC}

\section{Completion Report}

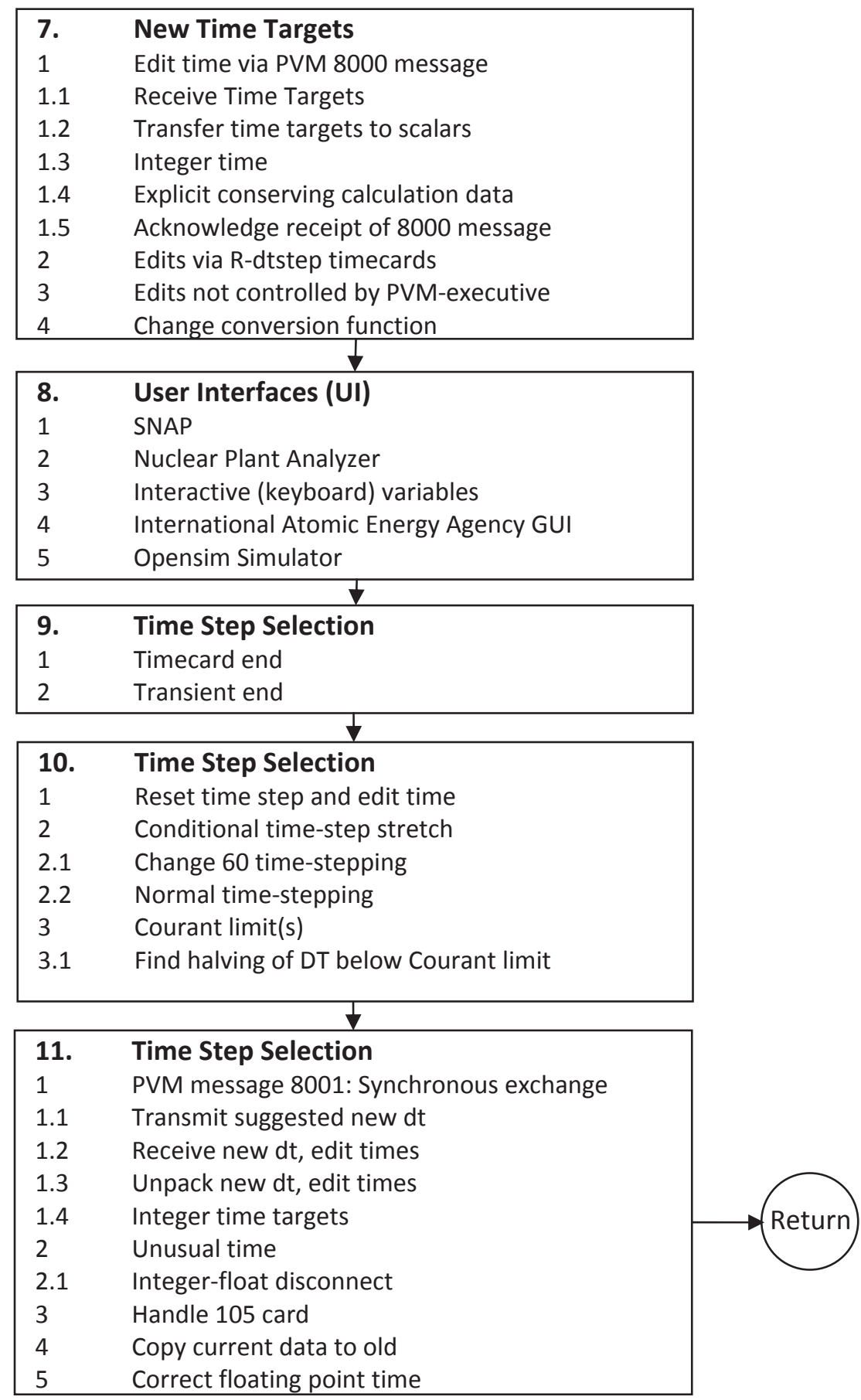

Figure 3b. Second portion of "Sectional Flowchart" for RELAP5-3D DTSTEP Functions

This sectional flowchart, when compared with the outline of DTSTEP in Section 6.1, shows code movement to recollect and unify functionality. It does not show jumps between sections. Figure 4 does. 


\section{Implementation of a New DTSTEP Algorithm \\ for use in RELAP5-3D and PVMEXEC}

Completion Report

Figure 4. DTSTEP High-level Flowchart showing jumps between sections.

In Figure 4, black is for normal flow and dark red jumps go to output. Other jumps shown lead to the subroutine exit. Jumps not shown are internal to a given flowchart section. In the initial DTSTEP coding, these included jumps around coding that did not belong to the section.

\subsection{Define, mnemonically rename, eliminate variables, and fix declarations, create integer timestep variables}

Before the conversion to integer timestepping could be undertaken effectively, workings of the DTSTEP subroutine had to be understood. Part of this was mapping and reorganizing the coding, but another part was deciphering the meaning of the variables and giving them more mnemonic names. This was undertaken simultaneously with the work of Sec 6.3.

All variables in DTSTEP relevant to the project were deciphered and documented in Table 4 . The meaning of variables such as gt19, iosini, nwqa, and $j$ (not a loop counter) then becomes clear for logic flow tracing, debugging, and conversion to integer timestepping.

Table 4. Data dictionary of all variables originally in DTSTEP

\begin{tabular}{|l|l|}
\hline \multirow{2}{*}{ Key: } & $\begin{array}{l}\text { Local variable names in lower case } \\
\text { GLOBAL variable names in UPPER case }\end{array}$ \\
\hline Name & Definition / Description \\
\hline af & time step Amplification Factor for time step (normally = 2.0). \\
\hline
\end{tabular}




\section{Implementation of a New DTSTEP Algorithm for use in RELAP5-3D and PVMEXEC}

\section{Completion Report}

\begin{tabular}{|c|c|}
\hline aflag & (G) true in dtstep when heat structures are advance checked in htadv \\
\hline CPUREM & CPU time REMaining - 5 elements from input file 105 -line. \\
\hline (1) & $\begin{array}{l}\text { Terminate transient immediately whenever CPUREM(1) > time remaining on CPU clock for } \\
\text { the run. }\end{array}$ \\
\hline (2) & $\begin{array}{l}\text { Terminate transient after successful time step whenever CPUREM(2) > time remaining on } \\
\text { CPU clock for the run. }\end{array}$ \\
\hline (3) & $\begin{array}{l}\text { CPU time remaining for the run. On input, it is the time allocated for the run. Zero means } \\
\text { no time limit. }\end{array}$ \\
\hline (4) & $\begin{array}{l}>0 \text { Turn on debug on this attempted advancement. } \\
=-1 \text { Write a dumpfile after advancement CPUREM( } 5) \& \text { quit. } \\
=-2 \text { Same as }-1 \text {, except don't quit, make } 2 \text { nd advancement and write a second dumpfile (for } \\
\text { comparison) \& quit. }\end{array}$ \\
\hline (5) & $>0$ Terminate code on this advancement. \\
\hline & = 0 Write dump file BEFORE starting transient \& terminate. \\
\hline curtmi & CURrent Time for next MInor edit. \\
\hline curtmj & CURrent Time for next MaJor edit. \\
\hline curtpl & CURrent Time for next PLot edit (time card option $\mathrm{d}=4$ \\
\hline curtrs & CURrent Time for next ReStart edit. \\
\hline curtex & CURrent Time for next EXplicit coupling data exchange. \\
\hline dtadj & the adjusted DT (doubled/halved). \\
\hline dtctr & ConTRol DT. \\
\hline dthyx & minimum of new adjusted HYdraulic DT and last time step value of $\mathrm{dtX}$. \\
\hline dtintv & DT INTerVal for minor/plot, major, or restart edits. \\
\hline dtmax_i & DTMAX from I-th timecard. \\
\hline dtmin_i & DTMIN from I-th timecard. \\
\hline dtrem & time REMaining on cpu clock for this problem. \\
\hline dtx & courant DT. \\
\hline gt14 & $\begin{array}{l}\text { Go To statement label } 14 \text {. } \\
\text { "Repeat time step with old values for dump file" flag. False initially. Set true if cpurei(4)==-2 }\end{array}$ \\
\hline gt19 & Go To statement label 19. set true if succes $==2$, false if $\mathrm{dt}<=\mathrm{dtmin} \_\mathrm{i}$ \\
\hline gt27 & $\begin{array}{l}\text { Go To statement label } 27 \text {. true if cpurem }(1) /=0 \& \text { dtrem }<=\text { cpurem(1) or succes }==6 \text { or help }<0 \\
\& /=-2 \text {. Set false initially } \& \text { when doing output }\end{array}$ \\
\hline gt38 & Go To statement label 38. help diagnostic step indicator. set true if help $<=2$. \\
\hline gt39 & Go To statement label 39. set false initially \& when doing output set true if done==-7. \\
\hline gt39a & $\begin{array}{l}\text { Go To statement label } 39 \text { Also. } \\
\text { Move (maybe) \& Exit Flag. set true if cpurei(4)>0 \& ncount >cpurei(5) }\end{array}$ \\
\hline gt100 & $\begin{array}{l}\text { Go To statement label 100. set true if: } \\
\text { succes }==0 \\
\text { succes }==1 \& \text { [iroute }==1 \text {.and. .not.btest(imdctl(1),8)) .or. .not.btest(tsc(it)\%tsppac,0)] }\end{array}$ \\
\hline gt101 & $\begin{array}{l}\text { Go To statement label } 101 . \text { small error time step doubler (if step was halved previously). } \\
\text { set false initially \& when .not.gt100 \& dt<=dtmin_i \& .not.gt19 } \\
\text { Set true if succes.le.1 \& [iroute.eq.1 .and. .not.btest(imdctl(1),8)) or } \\
\text {.not.btest(tsc(it)\%tsppac,0)] and .not.chngno(60) }\end{array}$ \\
\hline gtrtn & Go To ReTurN statement. true if done $/=0$ \\
\hline $\mathrm{i}$ & Index to current time step card. \\
\hline icard & fa-index of I-th (current) timeCARD. \\
\hline icoran & bin Index to use for COuRANt limit (normally 2). \\
\hline iecf & $\begin{array}{l}\text { Integer Edit Control Flag. packed word for minor edits, major edits, \& restarts edit control } \\
\text { flag, } 4 \text { bits, numbered from right. Also used as a counter in the courant limit calculations. }\end{array}$ \\
\hline
\end{tabular}




\section{Implementation of a New DTSTEP Algorithm for use in RELAP5-3D and PVMEXEC}

\section{Completion Report}

\begin{tabular}{|l|l|}
\hline \multicolumn{1}{|r|}{ Bit 1 } & $(=1)$ call rstrec for restart edits \\
\hline Bit 2 & $(=2)$ call majout for major edits \\
\hline Bit 3 & $(=4)$ call mirec for minor edits \\
\hline \multicolumn{1}{|c|}{ Bit 4 } & $(=8)$ call pltrec for plot edits. \\
\hline iosini & Indicator for On-line Semi-Implicit/Nearly-Implicit time advancement method. \\
\hline iplt & temporary storage for iecf. \\
\hline iprnt & PRINT Indicator, local value. \\
\hline it & Index of Time-step card \\
\hline ivskp2 & some bits out of print. \\
\hline j & pointer to next timestep card. \\
\hline last & true when LAST advancement for this time step card. \\
\hline nmon & Number of lines to write on MONitor before another label. \\
\hline numdt & NUMber of DTmax steps between timehy and the current edit times (curtmi, curtmj, curtrs). \\
\hline nwqa & pointer to second half of plot file scratch area. \\
\hline rf & Reduction Factor for time step (normally 0.5). \\
\hline ssdtim & Steady State TIMe step. \\
\hline stsold & time for next write to screen. \\
\hline SUCCES & Integer flag indicating SUCCESs of the advancement. \\
\hline & no need to repeat advancement with reduced time step \\
\hline 1 & excessive truncation error \\
\hline 2 & water property error \\
\hline 3 & non-diagonal matrix \\
\hline 4 & metal appears \\
\hline 5 & air appearance, velocity flip-flop, or water packing advancement with same time step size \\
\hline 6 & minimum volume of a variable volume \\
\hline timeend & END of requested TIME step. \\
\hline timlft & TIMe LeFT on this requested time step card, = timeend - timehy. \\
\hline
\end{tabular}

Note that this Table can be very useful when working with older versions of DTSTEP.

\subsubsection{Mnemonically rename variables}

Some of these variables, particularly the logical ones that started with "gt," were still unclear as defined above in terms of logical operations. These were given mnemonic names in Table 5. It is then clear from the name what the variable means.

Table 5. Mnemonic names for logical variables

\begin{tabular}{|l|l|l|}
\hline Name & Mnemonic Name & Meaning \\
\hline gt14 & ILoadOldTimeValues & Use subroutine MOVER to load old time values into current time arrays \\
\hline gt19 & IuserInterface & The user interface is activated \\
\hline gt27 & IEditThenQuit & Perform all output (plot, minor/major edits, restart) then quit \\
\hline gt38 & IRepeatWSameDt & $\begin{array}{l}\text { Repeat the advancement using the same timestep (happens when } \\
\text { there is water packing, velocity flip-flop, etc.) }\end{array}$ \\
\hline gt39 & IDoEdits & $\begin{array}{l}\text { Perform all selected output (among plot, minor/major edits, restart). } \\
\text { Note: Variable iecf hold the selection info. }\end{array}$ \\
\hline gt100 & IDoMsErrChk & Do mass error checking \\
\hline gt101 & IDoubleDt & Double the timestep \\
\hline
\end{tabular}




\title{
Implementation of a New DTSTEP Algorithm for use in RELAP5-3D and PVMEXEC
}

\author{
Completion Report
}

The use of camelback notation, where the first letter of each word in the variable name is capitalized, helps clarify what the name means at a glance.

Some of the variables initially eliminated were aflag, htimehy, nwqda, nwqdaa, and zeitmi. Many logical variables were eventually eliminated also.

\subsubsection{Fix the Declaration Section}

The declaration section was reorganized in three ways. First, all the conditional code marked with pre-compiler directives were put after unconditional code. Second, all integer statements were pulled together into a groups as were all real, logical, and character statements. Third, the variables on each declaration statement were alphabetized.

The unconditional declaration section was broken into two parts, the non-integer variables, and the integer variables. Each of these parts had a data dictionary from Tables 4, 5, and 6 . The only exceptions to this subdivision of declaration were references to new modules backmod and testmod, which were introduced for the integer algorithm and which must occur in the module section according to ANSI rules of Fortran 95.

\subsubsection{New Integer-Algorithm Variables}

Table 6 lists the local variables created to implement the integer timestepping.

Table 6. New integer timestepping variables created.

\begin{tabular}{|c|c|}
\hline Key: & Captialized letters in definition correspond to letters of name \\
\hline Variable Name & Definition \\
\hline curtmin & CURrenT MINimum of 3 time targets: minor \& major edits and restart \\
\hline diff & DIFFerence between two quantities. \\
\hline dtAdjTSave & $\begin{array}{l}\text { DT ADJusted Time SAVE. Time target for next advancement unless overridden by } \\
\text { Courant, stretch, or unusual time handling. }\end{array}$ \\
\hline dtmax_old & value of DTMAX from the OLD timecard, initialized to 0. \\
\hline dtmin_old & value of DTMIN from the OLD timecard, initialized to 0. \\
\hline dtratio & DTmax/dtmin RATIO \\
\hline dtsmallest & $\Delta \mathrm{t}_{\text {small }}$ from eq. $6 \mathrm{~b} ;$ mnemonic: SMALLEST usable DT. \\
\hline endlnterval & END points of timecard INTERVALs starting with the endpoint (which is normally 0). \\
\hline endold & ENDtime from OLD (previous) timecard \\
\hline endtimecard & ENDtime from current TIMECARD \\
\hline Icardbgn & Integer BEGinning time of current 30imecard. \\
\hline Icard_old & Index (fa) of previous time CARD. \\
\hline Idt & Integer timestep corresponding to real DT from eq. 8a. \\
\hline iduration & $\begin{array}{l}\text { Integer DURATION of the current timecard. } \\
\text { May differ from floating point duration because of unusual times. }\end{array}$ \\
\hline ledit & $\begin{array}{l}\text { Integer time target for EDITting (plot, minor, major, rest and possibly message- } \\
\text { exchange and end times) }\end{array}$ \\
\hline iendtran & Integer time target, END of TRANSIENT. \\
\hline lendtime & Integer time target, END of CURent time interval (card). \\
\hline
\end{tabular}


Implementation of a New DTSTEP Algorithm

Completion Report

\begin{tabular}{|l|l|}
\hline Inttwo & INTeger TWO, 8-byte constant value 2. \\
\hline Irall & Integer to Real difference relative test against dtsmALLest \\
\hline iredit & Integer Relative EDIT times. (minor, plot, major, restart, message-exchange) \\
\hline irtime & Integer Relative TIME. IRTIME .eq. ITIME - ICARDBGN. \\
\hline Itemp & Integer TEMPorary variable for calculating intermediate quantities \\
\hline itime & Integer equivalent of cumulative TIME, timehy \\
\hline Itimecard & Index, within endInterval, of the user's timecard that is in control \\
\hline kk & integer temporary variable for KalKulating intermediate quantities \\
\hline kNonInt & unusual handler linked-step number. mnemonic is: Kalculate NON-INTeger steps \\
\hline Istretch & Logical STRETCH logic active. \\
\hline Msgtim & MeSsaGe TIMe. Data transmitted via PVM messages. \\
\hline Nextdtmax & NEXT integer multiple of iDTMAX above cumulative time. \\
\hline Nhalvings & $\begin{array}{l}\text { Number of HALVingS necessary to reduce the maximum time step to as close as } \\
\text { possible to the minimum w/o going below it. }\end{array}$ \\
\hline Ntimecard & Number of TIMECARDs \\
\hline prevAdvTimecard & PREVious ADVancement's TIMECARD ordinal. \\
\hline supremum & the new timestep, which is a halving of dtmax, cannot exceed SUPREMUM. \\
\hline tyme & time (cumulative) calculated from integers. \\
\hline
\end{tabular}

\subsection{New Integer Timestepping Modules}

Two new modules were created for integer timestepping for RELAP5-3D DTSTEP. The modules are summarized in Table 7.

Table 7. New integer timestepping modules

\begin{tabular}{|l|l|}
\hline Name & Description \\
\hline Backmod & $\begin{array}{l}\text { Controls time step selection and frequency of output and plotting edits during transient } \\
\text { advancement. }\end{array}$ \\
\hline Testmod & $\begin{array}{l}\text { Test Matrix Module. Data for logic path input and logic path test processing. Its initial } \\
\text { design is for DTSTEP. }\end{array}$ \\
\hline
\end{tabular}

The variables of the Table 7 modules are summarized in Table 7a.

Table 7a. New integer timestepping modules

\begin{tabular}{|c|c|c|}
\hline Name & Variable & Description \\
\hline Backmod & IBackupOneDt & $\begin{array}{l}\text { Logical indicator for BACKing UP ONE DT (time-step). Important for New } \\
\text { Algorithm } 6 \text { - the unusual handler. } \\
\text {.true. means back-up was performed in DTSTEP and was not advanced. } \\
\text {.false. means back-up was not performed. } \\
\text { It is set in DTSTEP(LoadOldTimeValues-subr). Used in TRAN. }\end{array}$ \\
\hline Testmod & iTestDtstep & $\begin{array}{l}\text { Controls the Test Matrix Function in RELAP5-3D DTSTEP } \\
1=\text { force Courant } d t \text { halving } \\
2=\text { force halving of timestep } \\
3=\text { simulate PVM error condition } \\
4=\text { force minimal dt }\end{array}$ \\
\hline & ilowlimit & Activate the Test Matrix condition first on this timestep in subr. testDtst \\
\hline
\end{tabular}




\section{Implementation of a New DTSTEP Algorithm for use in RELAP5-3D and PVMEXEC}

\section{Completion Report}

\begin{tabular}{|l|l|l|}
\hline & iuplimit & Deactivate the Test Matrix condition on this timestep in subr. testDtstep. \\
\hline & testNo & In subr. rdbug, the Basic Test Case Number for the DTSTEP Test Matrix. \\
\hline & Aset & Test Matrix character strings identifying A-set as either A1 or A2. \\
\hline ctest & Complete description of the Basic Test Case to run: (Test\#)AABBCCDD \\
& & Test\# ranges from 1-17 \\
& AA ranges from A1 to A2 \\
& BB ranges from B1 to B3 \\
& CC ranges from C1 to C4 \\
& DD ranges from D1 to D7 \\
\hline & cbasic & Basic Test character identifier. Its values are "a1", "b1", "b2", "c1", "c2", \\
& "d1", "e1", "e2", "e3" \\
\hline
\end{tabular}

\subsection{Internal Subroutines}

Twenty internal subroutines were created. Some were abstracted from the code to clarify the logic flow of the main algorithm of DTSTEP. Others were created to have repeatable, reentrant code for use with the integer timstepping algorithm or for floating point or PVM-message operations. The subroutines are described in the following table.

Table 7b. New Internal Subroutines

\begin{tabular}{|c|c|c|}
\hline Name & Origin & Description \\
\hline astraTscCheck & Abstracted & $\begin{array}{l}\text { This normally inert coding belongs to the IAEA Graphical User Interface } \\
\text { (GUI) known as astra. }\end{array}$ \\
\hline doubleDt & Abstracted & $\begin{array}{l}\text { Only after an even number of advancements with the current timestep, } \\
\text { double the floating point timestep and halve variable nrepet, which } \\
\text { controls halving/doubling. (It has conditional experimental non- } \\
\text { halving/doubling coding for multiplying by 1.1, but this is almost never } \\
\text { used.) }\end{array}$ \\
\hline dthyCalc & Abstracted & $\begin{array}{l}\text { Reach the next floating point edit time target if it is within range if it is no } \\
\text { more than } 1.1^{*} \text { dtmax_i away from now, use one step: } \\
\text { - Timecard (or transient) endtime is the ultimate stretch target } \\
\text { - Never bypass the explicit exchange time. } \\
\text { Also calculates some legacy DTSTEP variables that are currently not in } \\
\text { use. }\end{array}$ \\
\hline dtoutput & Abstracted & $\begin{array}{l}\text { Perform all code output. Do edits according to the value of variable iecf. } \\
\text { Also do output for interfaces and to the screen. }\end{array}$ \\
\hline dtstat & Abstracted & $\begin{array}{l}\text { This coding collects the statistical measures of Figure 3a, section } 3 \\
\text { presented by major edits. }\end{array}$ \\
\hline dtterm & Abstracted & This coding collects the termination conditions of Figure 3a, section 2 . \\
\hline dt60select1 & Abstracted & $\begin{array}{l}\text { This belongs to the experimental non-halving/doubling coding that is } \\
\text { almost never used and is not part of the integer timestepping. }\end{array}$ \\
\hline editTime60 & Abstracted & $\begin{array}{l}\text { This belongs to the experimental non-halving/doubling coding that is } \\
\text { almost never used and is not part of the integer timestepping. }\end{array}$ \\
\hline exp_cpl_int & Abstracted & $\begin{array}{l}\text { Sends and receives the various types of explicit coupling messages. There } \\
\text { is logic to send the following four types of explicit messages: parallel } \\
\text { synchronous, parallel asynchronous, sequential synchronous and } \\
\text { sequential asynchronous. }\end{array}$ \\
\hline findHalving & bstracted & This coding implements New Algorithm 5. \\
\hline
\end{tabular}




\section{Implementation of a New DTSTEP Algorithm for use in RELAP5-3D and PVMEXEC}

\section{Completion Report}

\begin{tabular}{|c|c|c|}
\hline firstEntryOnly & Created & $\begin{array}{l}\text { Implements New Algortithm } 1 . \text { It creates array endInterval and initializes } \\
\text { it to timecard endtimes. It initializes many new scalar variables for the } \\
\text { integer timestepping. }\end{array}$ \\
\hline get800x & Abstracted & $\begin{array}{l}\text { For PVM Explicit messages } 8002 \text { through } 8005 \text {, listen for and receive } \\
\text { PVM-Executive's } 8000+x \text { message ( } x=2,3,4 \text {, or 5). This routine was } \\
\text { abstracted from } 4 \text { sections of nearly identical code in DTSTEP and reduced } \\
\text { to a single subroutine. } \\
\text { Check if "my task ID" is the SEND-task-ID in the message. } \\
\quad \text { If so, send my data (if any) to the appropriate task(s) and wait for a } \\
\text { reply. After a reply, possibly send again. } \\
\quad \text { If not, check if this task is to receive data from the SEND-task-ID. If so, } \\
\text { receive it, store it, and send acknowledgement. } \\
\text { Continue sending and receiving until all required data is sent. Then notify } \\
\text { PVM-Executive, data transfer is finished. PVM-Executive send } 8000+x \\
\text { message with sndtid==0 to indicate that message type is finished (and the } \\
\text { "Send or Receive Loop" may be exited). }\end{array}$ \\
\hline initTargets & Created & $\begin{array}{l}\text { Subroutine sets integer timestepping variables for the end of timecard } \\
\text { and end of transient. }\end{array}$ \\
\hline initIntTime & Created & Implements New Algorithm 4 and sets $\Delta \mathrm{t}_{1}$ to $\Delta \tau_{\max }$. \\
\hline intCardTargets & Created & $\begin{array}{l}\text { Implements New Algorithm 2, 3, and } 4 \text { either via direct calculation for } \\
\text { synchronous coupling, or else through calls to updateCardTarget. Also, } \\
\text { calculates integer equivalent to end of previous timecard and end of } \\
\text { current timecard. }\end{array}$ \\
\hline intTarg8000 & Abstracted & $\begin{array}{l}\text { This coding collects the Integer target operations conducted in the } 8000- \\
\text { section of dtstep, combining elements of New Algorithms } 2 \text { and } 4 . \text { Some } \\
\text { integer time quantity updates are performed only if coupling is } \\
\text { syncronous. }\end{array}$ \\
\hline LoadOIdTimeValues & Abstracted & $\begin{array}{l}\text { For the case of a code advancement back-up, move values from "old } \\
\text { time" arrays, that hold values from the previous advancement, into the } \\
\text { current time arrays. }\end{array}$ \\
\hline proc_8000 & Abstracted & $\begin{array}{l}\text { Processes the data associated with the } 8000 \text { message from PVMEXEC. It } \\
\text { determines if the message should be exchanged using the check } 8000 \\
\text { function from the shared module. If appropriate, the message is } \\
\text { received, the data are unpacked and the acknowledgement message is } \\
\text { sent back to PVMEXEC. }\end{array}$ \\
\hline proc_8001 & Abstracted & $\begin{array}{l}\text { Sends the } 8001 \text { message with a requested timestep size for } \\
\text { synchronously coupled calculations. The subroutine then receives the } \\
\text { floating point timestep size and the values for curtmi, curtmj and curtpl } \\
\text { from PVMEXEC. New values of iredit are calculated based on the } \\
\text { received edit times. }\end{array}$ \\
\hline updateCardTarget & Created & $\begin{array}{l}\text { If edit time has been reached, update edit times using user-specified } \\
\text { frequency. This implements New Algorithm 4, Eq. 7b for a single value of } \\
\text { j. }\end{array}$ \\
\hline
\end{tabular}

The following internal subroutines were abstracted by moving single-entry code and adjusting it to work correctly as a subroutine:

dthyCalc, dtterm, dtstat, LoadOldTimeValues, doubleDt, dtoutput, editTime60, Abstracted dt60select1, proc_8000, proc_8001. 


\section{Implementation of a New DTSTEP Algorithm for use in RELAP5-3D and PVMEXEC \\ Completion Report}

The following internal subroutines were constructed by abstracting coding used in more than one place to make multiply-called reentrant coding:

- findHalving - used in two places to find an acceptable $\Delta t$ at or below a target.

- Get800x - abstracted from 4 places to reduce coding into a single efficient routine.

The following internal subroutines were created to implement new integer algorithms or to incorporate integer algorithm coding. Subroutines dthyCalc and findhalving are listed here because they implement New Algorithms for integer timestepping.

dthyCalc, firstEntryOnly, initTargets, initIntTime, intCardTargets, updateCardTarget, findHalving, intTarg8000.

\subsection{Implementation in PVMEXEC DTSTEP}

The authors of PVMEXEC DTSTEP created it from a copy of the RELAP5-3D DTSTEP subroutine and adjusted as needed to function as the timestep controlling executive.

\subsection{Incorrect vestiges of RELAP5-3D in PVMEXEC DTSTEP}

Before the implementation of integer timestepping, PVM DTSTEP, the subroutine was studied and its functions identified. In the process, it was found that there were many comments, variables and some legacy code that had nothing to do with the function of the executive and were not used. Most were vestiges of RELAP5-3D DTSTEP. These confused the conversion to integer timestepping, development and debugging. There was also the possibility that they could inadvertently cause errors.

The following describes sections of the PVMEXEC DTSTEP subroutine that were either unnecessary, too complex, or perhaps incorrect (at least, it did not match the manual). Some comment statements were misleading or incorrect. Items are presented in the order that the statements or sections occur in base PVMEXEC DTSTEP, version 2.4.1.2. All were appropriately corrected as indicated with italicized remarks.

(1) On termination check (pvm_succes==6), DTSTEP does the following:

. set done $=-9$, iecf $=15$, gt $27=T$, go to 27

. set gt $27=$ gtrtn=F, iplt=iecf, skip msgs $8002-8005$ processing, gtrtn=T, go to 900

. skip: if gt $27==T$, go to 27 , finally RETURN becase gtrtn $==T$.

Since nothing is done with iecf, just return after done=-9.

(2) Section "Come here for a good advancement" is overly complex. Eliminate gt100 and label 801; just "go to 100" instead of 801.

(3) If timestep < minimum, do not stop; rather double timestep \& continue Actual statement: if (dt .le. dt_min(i)) go to 70

This is RELAP5-3D DTSTEP logic, but should be terminal in PVMEXEC.

(4) "Load old-time values for halved time-step" comment is meaningless.

Comment was removed.

(5) Failure conditions (pvm_success $>=2$ ) are checked AFTER timestep doubling.

This should be checked right after termination test (pvm_succes $==6$ ) 


\section{Implementation of a New DTSTEP Algorithm for use in RELAP5-3D and PVMEXEC}

Completion Report

(6) $\mathrm{k}=$ pvm_succes - 2 unneeded (from RELAP5-3D)

Just compare pvm_succes to 2 .

(7) The following comment is meaningless because there is no variable errmax.

"C If errmax is small, double halved time-step"

Comment was removed.

(8) Comment "Do requested edits" above statement 27 is misplaced.

What follows 27 is messages $8002-8005$ processing. Editing occurs after that the comment "send global print control to processes"

(9) Section "Finished with this transient?" should be in the advancement above the 8002-8005 section because messages $8002-8005$ do not change variable done.

(10) ** At the end of time interval, the last two steps are supposedly equal.

This condition is not enforced UNLESS nrepet $==0$ ( $d t==d t \max (i))$.

This is not what the manual says.

(11) The following comment is meaningless because there is no Courant limit in the Executive.

"c Set dtx to the minimum of courant, control, and $110 \%$ old value."

Removed comment.

(12) The following comment is meaningless because there is no 105 card in PVMEXEC:

"c Check if advancement is to be terminated by 105 card input."

Removed comment.

\subsection{Definitions, elimination of vestigal variables}

During the initial study of PVMEXEC DTSTEP, the variables were studied and defined to help understand the logic flow. Unnecessary variables and code were marked and eventually eliminated during the conversion process. Such variables are marked by asterisks in Table 8.

Table 8. PVMEXEC DTSTEP variables with potentially unneeded variables marked

\begin{tabular}{|l|l|}
\hline Name & Definition \\
\hline af & Amplification Factor for time step (normally = 2.0). \\
\hline contrl_timestep & CONTRoL TIMESTEP \\
\hline curctl & CURrent ConTroLling time step card number \\
\hline curtex & CURrent time target for EXplicit message \\
\hline curtmi & CURrent time target for MInor edit \\
\hline curtmj & CURrent time target for MaJor edit \\
\hline curtpl & CURrent time target for PLots \\
\hline curtrs & CURrent time target for RsStart \\
\hline done & O means not DONE, negative values indicate error conditions \\
\hline$d t$ & Delta Time, the current time step. \\
\hline$d t a d j$ & the ADJusted DT (doubled/halved). \\
\hline$d t c t r$ & ConTRol DT. it is a parameter = 10**75. Could be replaced. \\
\hline$d t$ control & $\begin{array}{l}\text { DT CONTROL information, an array with length n_timesteps, its bits control the time } \\
\text { stepping for each timecard. }\end{array}$ \\
\hline$d t h y$ & HYdraulic DT. minimum of new adj dt and last time step value of dtx. \\
\hline$* * d t h y x$ & HYdraulic DT eXtra copy. not used. Should be deleted. \\
\hline$* * d t h t$ & HeaT DT. set but never used. Should be deleted. \\
\hline$* * d t i n t v$ & DT INTeRval for minor/plot, major, or restart edits. \\
\hline
\end{tabular}


Completion Report

\begin{tabular}{|c|c|}
\hline$* *$ dtrem & Delta Time (cpu_limit - timehy) REMaining on cpu clock for this problem. \\
\hline dtx & courant DT. \\
\hline$* * d t x m d t$ & ?? declared in DTSTEP but not used. Should be removed. \\
\hline edittime & minimum of approaching EDIT TIME targets. \\
\hline endtime & END TIME of current timecard. right endpt of time interval \\
\hline end_time & array of END TIMEs of all the timecards. \\
\hline$* *$ exflag & EXplicit FLAG. \\
\hline exp_cpl_freq & FREQuency of EXPlicit CouPLing message exchanges. \\
\hline fail & FAILure indicater. true means code failure; quit. \\
\hline$* *$ flag & $\begin{array}{l}\text { true if we still need to do some steps to get to dtmax. } \\
* * \quad \text { always has value "nrepet.eq.0". Should be replaced. }\end{array}$ \\
\hline gt100 & $\begin{array}{l}\text { Go To 100. true if-and-only-if pvm_succes .eq. } 0 \\
\text { At label } 100 \text { "if errmax small, double halved time-step" }\end{array}$ \\
\hline gt101 & $\begin{array}{l}\text { Go To } 101 . \text { when true, decrement nrepet. } \\
\text { Label } 101 \text { is within the Label } 100 \text { section. }\end{array}$ \\
\hline$* *$ gt14 & Go To 14. ALWAYS FALSE and should be removed. \\
\hline$* *$ gt19 & Go To 19. true only if pvm_succes .eq. 2 . \\
\hline gt27 & $\begin{array}{l}\text { Go To } 27 . \text { true only if pvm_succes .eq.6. } \\
\text { Label } 27 \text { starts the "Do requested Edits" section. }\end{array}$ \\
\hline$* *$ gt38 & Go To 38. ALWAYS FALSE and should be removed. \\
\hline$* * \mathrm{gt} 39$ & Go To 39. ALWAYS FALSE and should be removed. \\
\hline **gt39a & Go To 39. ALWAYS FALSE and should be removed. \\
\hline gtrtn & Go To ReTurN \\
\hline$* *$ help & HELP variable from RELAP5-3D. declared in DTSTEP but used nowhere in PVMEXEC. \\
\hline$* *$ htimehy & from RELAP5-3D, declared in DTSTEP but used nowhere in PVMexec. \\
\hline$* *$ itimehy & from RELAP5-3D, declared in DTSTEP but used nowhere in PVMexec. \\
\hline$* *_{\mathrm{i}}$ & index to current time step card. Same as curctl. \\
\hline$* *$ icard & from RELAP5-3D, declared in DTSTEP but used nowhere in PVMexec. \\
\hline$* *$ icoran & from RELAP5-3D, declared in DTSTEP but used nowhere in PVMexec. \\
\hline iecf & $\begin{array}{l}\text { packed word for minor edits, plots, major edits, restarts edit control flag, } 4 \text { bits, } \\
\text { numbered from right } \\
1(=1) \text { call rstrec for restart edits } \\
2(=2) \text { call majout for major edits } \\
3(=4) \text { call mirec for minor edits } \\
4(=8) \text { call pltrec for plot edits. }\end{array}$ \\
\hline info & integer*4, INFOrmation necessary for PVM. \\
\hline iplt & temporary storage for iecf? \\
\hline$* *$ iprnt & never used and should be eliminated. local value of print. \\
\hline$* *$ iv & from RELAP5-3D, declared in DTSTEP but used nowhere in PVMexec. \\
\hline$* *$ ivskp2 & from RELAP5-3D, declared in DTSTEP but used nowhere in PVMexec. \\
\hline$* *$ iwrd8 & from RELAP5-3D, declared in DTSTEP but used nowhere in PVMexec. \\
\hline j & pointer to next timestep card. \\
\hline last & true if this is the last adv. for this time step card. \\
\hline lexplt & Logical, EXPLiciT message exchange control \\
\hline Iminor & Logical, MINOR edit message exchange control \\
\hline
\end{tabular}


Completion Report

\begin{tabular}{|c|c|}
\hline Imajor & Logical, MAJOR edit message exchange control \\
\hline Iplot & Logical, PLOT message exchange control \\
\hline Irst & Logical, ReSTart message exchange control \\
\hline$* *$ messg & from RELAP5-3D, declared in DTSTEP but used nowhere in PVMexec. \\
\hline mip_freq & MInor edit/Plot FREQuency (number of dt_max timesteps major edits.) \\
\hline mj_freq & MaJor edit FREQuency (number of dt_max timesteps between major edits.) \\
\hline msgtag & MeSsaGe TAG for pvm. \\
\hline$* *$ nbyte & declared in DTSTEP but used nowhere in PVMexec. \\
\hline ncount & Number or COUNT of time step advancements \\
\hline nitem & integer*4, Number of ITEMs in a message. \\
\hline$* *$ nmechk & No Mass Error CHecK, from RELAP5-3D. set but never used and should be removed. \\
\hline$* *$ nmon & declared in DTSTEP but not used. \\
\hline nrepet & Number of REPEaTs of current time step to reach next multiple of DTMAX. \\
\hline$* *$ nstsp & $\begin{array}{l}\text { Set but never used. Should be removed. set to zero and incremented or } \\
\text { decremented but not output or in any test or calculation.) }\end{array}$ \\
\hline$* *$ nwqa & declared in DTSTEP but not used anywhere in PVMexec. \\
\hline$* *$ nwqda & declared in DTSTEP but not used anywhere in PVMexec. \\
\hline$* *$ nwqdaa & declared in DTSTEP but not used anywhere in PVMexec. \\
\hline n_exsync & Number of EXplicit SYNChronously coupled processes \\
\hline n_exasync & Number of EXplicit ASYNChronously coupled processes \\
\hline n_excsync & Number of EXplicit SYNChronous sequential coupled processes \\
\hline n_excsync & Number of EXplicit ASYNChronous sequential coupled processes \\
\hline n_proc & Number of coupled PROCesses \\
\hline n_synch & Number of SYNChronously coupled processes \\
\hline n_timesteps & Number of TIMESTEP cardS \\
\hline$* *$ numdt & $\begin{array}{l}\text { integer NUMber of DTmax steps between timehy and the current edit times (curtmi, } \\
\text { curtmj, curtrs). never used. Should be removed. }\end{array}$ \\
\hline **prevnd & PREVious time step card eND time. Set but never used. Should be removed. \\
\hline procdt & The proposed DT by the synchronously coupled PROCesses \\
\hline proc_rpt & $\begin{array}{l}\text { list of PROCesses RePorT statuses } \\
0 \text { not reported } \\
1 \text { reported }\end{array}$ \\
\hline proc_sync & list of PROCesses coupled SYNChronously \\
\hline pvmdt & $\begin{array}{l}\text { PVMexec selected DT = minimum value of all time steps proposed by synchronously } \\
\text { coupled processes. }\end{array}$ \\
\hline$* *$ pvmint4 & INTEGER4 \\
\hline **pvmreal8 & REAL8 \\
\hline pvm_succes & $\begin{array}{l}\text { SUCCESs of the PVM coupled calculation at current step. } \\
0 \text { means continue } \\
1 \text { means running at minimum time step is okay } \\
x \text { for } 1<x<6 \text { are various time step controls } \\
6 \text { means stop the coupled calculation }\end{array}$ \\
\hline $\mathrm{rf}$ & Reduction Factor for time step (normally 0.5 ). \\
\hline \multirow[t]{2}{*}{ rpt_stat } & RePorT STATus of all processes \\
\hline & $0=$ not all process reported \\
\hline
\end{tabular}




\section{Implementation of a New DTSTEP Algorithm for use in RELAP5-3D and PVMEXEC \\ Completion Report}

\begin{tabular}{|l|l|}
\hline & 1 = all processes reported \\
\hline rst_freq & ReSTart FREQuency (number of dt_max timesteps between restarts.) \\
\hline skipt & SKIP certain coding when True (.true. when the calculation is a restart) \\
\hline sndtid & Task IDentification to SeND \\
\hline stride & integer*4, STRIDE (skip factor) through the data. \\
\hline$* *$ stsold & declared in DTSTEP but used nowhere in PVMexec. \\
\hline tid & integer*4, Task IDentifier. \\
\hline timehy & cumulative TIME HYdraulic. \\
\hline$* *$ time4 & declared but never used. \\
\hline$* *$ timlft & $\begin{array}{l}\text { time left on this time step card, tpsend(i)-timehy. Set but never used. Should be } \\
\text { removed. }\end{array}$ \\
\hline
\end{tabular}

Of the variables marked with ${ }^{* *}$ in Table 8 as being potentially unneeded at the early stage of the project, 34 were eliminated. They are:

dtht, dthyx, dtintv, dtrem, dtxmdt, exflag, flag, gt14, gt19, gt38, gt39, gt39a, help, htimehy, icard, icoran, iprnt, itimehy, iv, ivskp2, iwrd8, messg, nbyte, nmechk, nmon, nstsp, numdt, nwqa, nwqda, nwqdaa, prevnd, stsold, time4, timlft

The three, that were kept because they were found to be necessary, are: i, pvmint4, pvmreal8

\subsection{Reworking source code of PVMEXEC DTSTEP}

The source code was made more readable by eliminating unused variables and code, inserting a data dictionary, inserting numerous, outline-style comments, and regrouping sections of code. This aided in the conversion and debugging processes.

\subsubsection{Deleted source code from PVMEXEC DTSTEP}

Coding that was removed includes:

- All declarations of unused variables that were eliminated in Sec. 7.2

- All statements that used unused variables that were eliminated in Sec. 7.1

- Some code that was unreachable because some logical variables were always false

\subsubsection{Declarations in PVMEXEC DTSTEP}

The declaration statements were grouped as follows:

- modules in alphabetical order (except for machine)

- other global variables (include statements and common block dtstepc)

- local variables.

Among global and local variables, the declarations are grouped by type; the groups are ordered:

- Real declarations

- Integer declarations

- Logical

Variables were alphabetized on declaration statement to aid in finding them, except for the common statements which induces an order. 


\section{Implementation of a New DTSTEP Algorithm for use in RELAP5-3D and PVMEXEC \\ Completion Report}

The local declaration section was further subdivided into original and integer timestepping variables. The original section was divided into active and eliminated. The declaration of all variables that were eliminated in Sec. 7.1 were collected and marked as junk. These were later removed altogether after verifying that they had no impact on code performance.

All unused sections of declarations marked with pre-compiler directives were eliminated. These three sections are:

$$
\text { bufr, cmprs, npa }
$$

All declarations of unused variables, those that were marked in Table 7 with asterisks were also removed.

\subsection{Internal documentation in PVMEXEC DTSTEP}

An outline style of numbered comments, using the 1, 1.1, 1.1.1, system for subsections was introduced to break out major sections and progressively minor subsections. Explanations of operations were expanded from 0-2 lines to whatever amount was deemed necessary to explain a section to a developer who is completely unfamiliar with DTSTEP. This was helpful in the development and debugging stages.

During the development and debugging, sometimes the comments became incorrect. Attempts were made to keep them correct; however, this was not fully successful.

\subsubsection{Data dictionary}

There are two data dictionaries:

- original variables

- integer timestepping variables.

The variables are listed in alphabetical order with its definition using a capitalization key to indicate the mnemonics of the name.

The original variable data dictionary was constructed from Table 8. Those variables that are not marked with asterisks were eventually the only ones remaining in the data dictionary. The integer timestepping variables data dictionary follows the declaration for the original variables in DTSTEP.

\subsection{Integer Timestepping in PVMEXEC DTSTEP}

The implementation involved creating and documenting the new variables for timestepping, coding the integer timestepping New Algorithms, and breaking out internal subroutines. The latter is covered in the next section.

\subsubsection{Local integer timestepping variables}

The new variables introduced to PVMEXEC DTSTEP to implement the integer timestepping are given in Table 9.

Table 9. Integer Timestepping Local Variables in PVMEXEC DTSTEP

\begin{tabular}{|l|l|}
\hline$d t s a v$ & DT SAVe. Copy of dt made before special timecard endtimes \\
\hline$d t$ targ & DT TARGet. $d t$ made to handle special timecard endtimes to override pvmdt as \\
\hline
\end{tabular}


Completion Report

\begin{tabular}{|l|l|}
\hline & requested by coupled codes. \\
\hline icardkind & Integer timeCARD-defined frequency of given KIND of edit (plot, restart, etc) \\
\hline idtsav & Integer DT SAVe. Copy of idt made before special timecard endtimes \\
\hline idt_targ & $\begin{array}{l}\text { Integer DT TARGet. idt made to handle special timecard endtimes used to override } \\
\text { pvmdt as requested by coupled codes. }\end{array}$ \\
\hline iredit & Integer Relative EDIT time, $\tau_{\mathrm{E}, \mathrm{k}}$ of Eq. (8b). \\
\hline itime & Integer cumulative TIME, $\mathrm{T} / \Delta \mathrm{t}_{\text {small. }}$ \\
\hline itmp & Integer TeMPorary \\
\hline itsav & $\begin{array}{l}\text { Integer Time (cumulative) SAVe. Copy of timehy made before handling special } \\
\text { timecard endtimes }\end{array}$ \\
\hline Iadd(5) & $\begin{array}{l}\text { Logical Array of Dump time inDicators. (Not used) } \\
(1) \text { Iminor, (2) Iplot, (3) Imajor, (4) Irst, (5) lexplt }\end{array}$ \\
\hline IBackupOneDt & $\begin{array}{l}\text { Logical indicator to BACKUP ONE DT (time-step). } \\
\text { true. means do a backup }\end{array}$ \\
\hline Irestore & $\begin{array}{l}\text { Logical RESTORE flag. when TRUE it means that dt and idt are to be restored to } \\
\text { dtsav and idtsav and ovderride the pvmdt requested by the coupled codes. }\end{array}$ \\
\hline supremum & the new timestep, which is a halving of dtmax, cannot exceed SUPREMUM. \\
\hline tdiff & $\begin{array}{l}\text { Time DIFFerence. Difference between floating point time and time calculated from } \\
\text { integers. }\end{array}$ \\
\hline tmpmax & integer TeMPorary MAXimum used to calculate idtmax. \\
\hline tsav & $\begin{array}{l}\text { Time (cumulative) SAVe. Copy of timehy made before handling special timecard } \\
\text { endtimes. }\end{array}$ \\
\hline tyme & Ti(Y)ME calculated from integers for diagnostics. \\
\hline
\end{tabular}

\subsubsection{Module integer timestepping variables}

A new module for integer timestepping variables was created called TARGETMOD. It contains data for the PVM Executive's integer time targets that specify the following:

(1) End of transient

(2) End of time for the current timecard

(3) Explicit message exchange time

(4) Major edit time

(5) Minor edit time

(6) Plot time

(7) Restart dump time

The module variables are given in Table 10.

Table 10. Integer Timestepping Local Variables in TARGETMOD

\begin{tabular}{|l|l|}
\hline dtsmallest & SMALLEST allowable value of DT, $\Delta \mathrm{t}_{\text {small }}$ from New Algorithm 1, Eq. 6b \\
\hline endInterval & $\begin{array}{l}\text { END time of a time INTERVAL (right endpoint). } \\
\text { endInterval( } 0 \text { ) = "start time of case" }\end{array}$ \\
\hline hitend & $\begin{array}{l}\text { HIT the END of timecard. true means close enough to hit end by adjusting } \mathrm{dt} \\
\text { (compressing or stretching up to } 110 \% \text { ) }\end{array}$ \\
\hline icardbgn & Integer BeGiNning time of current timeCARD in terms of dtsmallest. $\mathrm{T}_{\mathrm{C}-1} / \Delta \mathrm{t}_{\text {small. }}$ \\
\hline icardend & Integer timecard END time of current CARD in terms of dtsmallest. $\left(\mathrm{T}_{\mathrm{C}}-\mathrm{T}_{\mathrm{C}-1}\right) / \Delta \mathrm{t}_{\text {small. }}$ \\
\hline icardtime & Integer TIME accumulated since start of current timecard. $\tau_{\mathrm{C}, \mathrm{i}}$ of Eq. (7). \\
\hline idt & Integer DT, $\Delta \tau_{\mathrm{i}}$ in New Algorithm 4, Eq. (8a) \\
\hline
\end{tabular}


Implementation of a New DTSTEP Algorithm

\section{Completion Report}

\begin{tabular}{|c|c|}
\hline & \# of Herz or relap5-cycles that equal dt. \\
\hline idtadj & Integer DT ADJustment. Not used (yet) \\
\hline iduration & $\begin{array}{l}\text { Integer DURATION of the current timecard = icardend - icardbgn. } \\
\text { May differ from floating point duration because of unusual times. }\end{array}$ \\
\hline iloc & i-value LOCating the minimum dt_min among timecards \\
\hline idtmax & $\begin{array}{l}\text { Integer largest value of DTMAX. } \\
\Delta \tau_{\max }=2^{H} \text { in New Algorithm 1, Eq. (6a) }\end{array}$ \\
\hline iedit & Integer EDIT time \\
\hline iendtime & Integer END TIME for the entire transient \\
\hline iexplfrq & Integer EXPLicit FReQuency. exp_cpl_freq \\
\hline iexpltime & Integer EXPLicit TIME. relap5-cycle for exchanging explicit coupling data \\
\hline iloc & I-value LOCating the minimum dt_min among timecards. \\
\hline imajorfrq & Integer MAJOR FReQuency. mj_freq \\
\hline imajortime & Integer MAJOR TIME. relap5-cycle for next major edit \\
\hline iminorfrq & Integer MINOR FReQuency. mip_freq \\
\hline iminortime & Integer MINOR TIME. relap5-cycle for next minor edit \\
\hline iplotfrq & Integer PLOT FReQuency. mip_freq \\
\hline iplottime & Integer PLOT TIME. relap5-cycle for next plot \\
\hline irestfrq & Integer RESTart FReQuency. rst_freq \\
\hline iredit & $\begin{array}{l}\text { Integer Relative EDIT times. These are restarted at the beginning of each timecard. } \\
1=\text { minor edit, } 2=\text { plot, } 3=\text { major edit, } 4=\text { restart, } 5=\text { explicit coupling message time }\end{array}$ \\
\hline kk & integer temporary variable for KalKulating intermediate quantities \\
\hline nhalvings & $\begin{array}{l}\text { Number of HALVINGS that reduces DTMAX to dtsmallest. } \\
\mathrm{H} \text { in New Algorithm 1, Eq. } 6\end{array}$ \\
\hline prevAdvTimecard & index among user TIMECARDs of the PREVious ADVancement \\
\hline spstat & $\begin{array}{l}\text { SPecial STATus. Indicates status of processing a special endtime. } \\
0 \text { not at an unusual time target, proceed normally. } \\
1 \text { complete final step of an unusual time target; the first step (spstat.eq.2) was } \\
\text { taken on the previous step. Hereafter, time is at a normal halving/doubling } \\
\text { point. } \\
2 \text { timestep exactly reaches unusual time target. } \\
3 \text { (may not be taken) timestep is half the distance to the unusual time target. }\end{array}$ \\
\hline iresttime & Integer RESTart TIME. relap5-cycle for next restart file write \\
\hline itime & Integer TIME \\
\hline
\end{tabular}

\subsection{Subroutines introduced for restructuring}

As with the creation of the PVMEXEC DTSTEP from a copy of the RELAP5-3D DTSTEP subroutine, the internal subroutines were initially copies of those in RELAP5-3D DTSTEP. They were then adjusted as needed. This exactly follows the original methodology employed by the authors of the PVM Coupling coding.

A significant effort was made to restructure the code during the resolution of this issue. Primarily, this has been accomplished by taking the previous $d t s t e p$ subroutine and reducing it to the high-level logic path with a significant number of "contained" subroutines. This restructuring is most noticeable in the PVMEXEC $d$ tstep subroutine. While the restructuring of the code did not solve any problems, it did provide additional clarity as to the overarching code logic, which did help in providing a better understanding of the algorithm which ultimately led 


\title{
Implementation of a New DTSTEP Algorithm for use in RELAP5-3D and PVMEXEC
}

\author{
Completion Report
}

to a successful implementation of the integer-based timestep size selection and advancement algorithm.

Fourteen internal subroutines were created. Some were abstracted from the code to clarify the logic flow of the main algorithm of DTSTEP. Others were created to have repeatable, reentrant code for use with the integer timstepping algorithm or for floating point or PVM-message operations. The subroutines are described in the following table.

Table 7a. New Internal Subroutines

\begin{tabular}{|c|c|c|}
\hline Name & Origin & Description \\
\hline firstEntryOnly & Created & $\begin{array}{l}\text { On the very first entry to DTSTEP (and not thereafter), perform } \\
\text { initializations and allocations. Find minimum time-step on all timecards. } \\
\text { Differences with RELAP5-3D counterpart, firstEntryOnly: } \\
\text { No endInterval array and fewer initializations. }\end{array}$ \\
\hline $\begin{array}{l}\text { explicit_async_- } \\
\text { parallel_exchange }\end{array}$ & Abstracted & $\begin{array}{l}\text { This subroutine determines if this is the appropriate time to perform an } \\
\text { explicit, parallel, asynchronous data exchange between any of the } \\
\text { coupled codes. If it is, each of the codes that should be sending data is } \\
\text { notified. The subroutine then waits for acknowledgement that all of the } \\
\text { receivers have obtained all of their data. Finally, this subroutine sends } \\
\text { the "all clear" message to all of the explicit, asynchronous, parallel } \\
\text { coupled jobs to permit them to resume their calculations. }\end{array}$ \\
\hline $\begin{array}{l}\text { explicit_async_- } \\
\text { seq_exchange }\end{array}$ & Abstracted & $\begin{array}{l}\text { This subroutine determines if this is the appropriate time to perform an } \\
\text { explicit, sequential, asynchronous data exchange between any of the } \\
\text { coupled codes. If it is, each of the codes that should be sending data is } \\
\text { notified. The subroutine then waits for acknowledgement that all of the } \\
\text { receivers have obtained all of their data. Finally, this subroutine sends } \\
\text { the "all clear" message to all of the explicit, asynchronous, parallel } \\
\text { coupled jobs to permit them to resume their calculations. }\end{array}$ \\
\hline $\begin{array}{l}\text { explicit_sync_ } \\
\text { parallel_exchange }\end{array}$ & Abstracted & $\begin{array}{l}\text { If a simulation includes explicit, synchronous, parallel coupling, each of } \\
\text { the codes that should be sending data is notified. The subroutine then } \\
\text { waits for acknowledgement that all of the receivers have obtained all of } \\
\text { their data. Finally, this subroutine sends the "all clear" message to all of } \\
\text { the explicit, asynchronous, parallel coupled jobs to permit them to } \\
\text { resume their calculations. }\end{array}$ \\
\hline $\begin{array}{l}\text { explicit_sync_- } \\
\text { seq_exchange }\end{array}$ & Abstracted & $\begin{array}{l}\text { If a simulation includes explicit, synchronous, sequential coupling, each } \\
\text { of the codes that should be sending data is notified. The subroutine then } \\
\text { waits for acknowledgement that all of the receivers have obtained all of } \\
\text { their data. Finally, this subroutine sends the "all clear" message to all of } \\
\text { the explicit, asynchronous, parallel coupled jobs to permit them to } \\
\text { resume their calculations. }\end{array}$ \\
\hline findHalving & Abstracted & $\begin{array}{l}\text { This coding implements New Algorithm 9. } \\
\text { Moved into the shared module, idtmod; see Section 8.0. }\end{array}$ \\
\hline intCardTargets & Created & 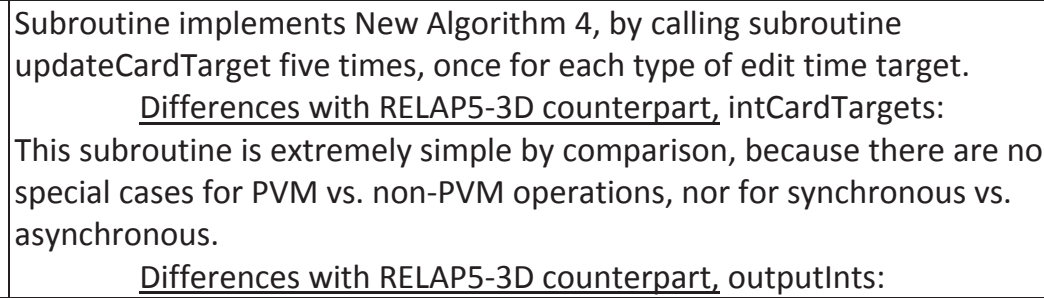 \\
\hline
\end{tabular}




\title{
Implementation of a New DTSTEP Algorithm for use in RELAP5-3D and PVMEXEC
}

\author{
Completion Report
}

\begin{tabular}{|c|c|c|}
\hline & & This one uses array iredit rather than mnemonically named scalars. \\
\hline intDtMax & Created & This function subprogram implements New Algorithm 1. \\
\hline outputInts & $\begin{array}{l}\text { Abstracted } \\
\text { Deprecated }\end{array}$ & $\begin{array}{l}\text { Subroutine implements New Algorithm 4, by calling subroutine } \\
\text { updatelntTarg five times, once for each type of edit time target, using } \\
\text { mnemonically named scalars. } \\
\text { Differences with RELAP5-3D counterpart, intCardTargets: } \\
\text { This subroutine is extremely simple by comparison, because } \\
\text { there are no special cases for Pvm vs. non-PVM operations, nor } \\
\text { for synchronous vs. asynchronous. } \\
\text { This subroutine was replaced by intCardTargets below. }\end{array}$ \\
\hline process_8000 & Abstracted & $\begin{array}{l}\text { This subroutine processes the data associated with } 8000 \text { message. It } \\
\text { determines if the message should be exchanged using the check_ } 8000 \\
\text { function from the shared module. If appropriate, the message data are } \\
\text { packed and sent to the appropriate coupled code. The } \\
\text { acknowledgement message for message tag } 8000 \text { is also received. }\end{array}$ \\
\hline rec_8001 & Abstracted & $\begin{array}{l}\text { This subroutine receives the } 8001 \text { message, which contains the } \\
\text { requested timestep size, from each of the codes that are synchronously } \\
\text { coupled. }\end{array}$ \\
\hline timeCardlnit & Created & $\begin{array}{l}\text { Subroutine called at the end of timecards to implement New Algorithm } \\
2,3 \text {, and } 4 \text { in setting up next timecard's control. It finds dtsmallest from } \\
\text { the next dtmax and dtmin, resets integer timecard time to zero, and } \\
\text { reset all integer time targets. } \\
\quad \text { Differences with RELAP5-3D counterparts, initIntTime \& } \\
\text { intCardTargets: } \\
\text { It performs many more initializations than initIntTime. Unlike } \\
\text { IntCardTargets does not call updateCardTarget to initialize time targets; } \\
\text { it also uses mnemonic scalars also for integer time targets. }\end{array}$ \\
\hline updateCardTarget & Created & $\begin{array}{l}\text { If edit time has been reached, update edit times using user-specified } \\
\text { frequency (RELAP5-3D Manual Volume 2, Appendix A). This implements } \\
\text { New Algorithm 4, Eq. (8b) for a single value of j. Also implements the } \\
110 \% \text { stretch logic of Sec. 5.4. } \\
\text { Differences with RELAP5-3D counterpart, updateCardTarget: } \\
\text { The user-specified frequency can be zero; so there is special coding to } \\
\text { handle that. Also, the stretch logic is not present in the counterpart. }\end{array}$ \\
\hline updatelntTarg & \begin{tabular}{|l|} 
Created \\
Deprecated
\end{tabular} & $\begin{array}{l}\text { Subroutine implements New Algorithm 4, Eq. (8b) for one value of j. It } \\
\text { converts a timecard edit frequency (RELAP5-3D Manual Volume 2, } \\
\text { Appendix A) to an integer frequency, ifreq, carefully. } \\
\text { Differences with RELAP5-3D counterpart, updateCardTarget: } \\
\text { It performs the rounding conversion that RELAP5-3D DTSTEP } \\
\text { internal subroutine intCardTargets does in addition to the } \\
\text { minimization adjustment of updateCardTarget. } \\
\text { This subroutine was replaced by updateCardTarget below. }\end{array}$ \\
\hline
\end{tabular}

Among these subroutines, most were created specifically to implement the integer algorithm.

\subsection{Shared Module - idtmod}

The PVMEXEC and RELAP5-3D computer programs both have similar tasks associated with the selection of timestep sizes to hit certain specified communication points. However, many 


\title{
Implementation of a New DTSTEP Algorithm for use in RELAP5-3D and PVMEXEC
}

\author{
Completion Report
}

different routines or techniques were used to perform the same task. The use of different implementations of what should have been the same logic created scenarios where the inconsistencies, while maybe minor (including different order-of-arithmetic issues), were sufficient to preclude proper execution of the problems. To remedy this, a shared module, idtmod, was created. This module contains both data and sub-programs that are needed by both PVMEXEC and RELAP5-3D.

A number of quantities and functions are exactly the same between the two subroutines RDTSTEP and P-DTSTEP. Many variables and utility subroutines were identified, renamed to be consistent between the DTSTEP subroutines, and collected into the shared module.

\subsection{Data}

The shared module has been developed to contain a small amount of data that is needed by the integer-based algorithm. The variables that are included in the module are detailed in Table 11.

Table 11. Integer Timestepping Local Variables in TARGETMOD

\begin{tabular}{|c|c|}
\hline \multicolumn{2}{|l|}{ Logicals } \\
\hline exp_cpl & Specify if EXPlicit CouPLing is active \\
\hline $\mathrm{Idbg}$ & Logical DeBuG flag. When true, it activates additional debug print statements \\
\hline \multicolumn{2}{|l|}{ Integers } \\
\hline i8kind & Integer 8-byte KIND. Specifies 64 bit integer values as need by the algorithm \\
\hline qdkind & QuaD-precision KIND. Specifies 128 bit floating point values \\
\hline dpkind & Double-Precision KIND. Specifies 64 bit floating point values \\
\hline dbgf & DeBuG File. The unit number used for the debug print statement \\
\hline idt & $\begin{array}{l}\text { Integer Delta-T. Representation of the timestep size. For normal timesteps, } \\
\mathrm{dt=idt*dtsmallest}\end{array}$ \\
\hline idtmax & Integer DT MAXimum. Integer representation of the maximum timestep size \\
\hline itime & Integer cumulative TIME. Integer counter for elapsed time \\
\hline iendtime & Integer END TIME of the timecard. \\
\hline iendtran & Integer END of TRANsient. Integer representation of the transient end time \\
\hline icardbgn & $\begin{array}{l}\text { Integer timeCARD BeGiN time. Integer representation for the start time of the } \\
\text { current timecard }\end{array}$ \\
\hline icardend & $\begin{array}{l}\text { Integer timeCARD END time. Integer representation for the time equal to the end of } \\
\text { the current time card }\end{array}$ \\
\hline irtime & $\begin{array}{l}\text { Integer Relative TIME. Integer representation of time elapsed from start of current } \\
\text { timecard }\end{array}$ \\
\hline iduration & Integer DURATION. number of timestep of size dtsmallest in the current time card \\
\hline iredit(5) & Integer Representation of EDIT times \\
\hline iedit & Integer representing the nearest EDIT point \\
\hline \multicolumn{2}{|c|}{ Extended Precision Reals (128-bit floating point) } \\
\hline qdtsmallest & $\begin{array}{l}\text { QuaD-precision SMALLEST attainable DT. Floating point timestep size that is greater } \\
\text { than DTMIN and is determined via Eq (1) }\end{array}$ \\
\hline
\end{tabular}




\section{Implementation of a New DTSTEP Algorithm

\section{Completion Report}

\begin{tabular}{|l|l|}
\hline dtsmallest & $\begin{array}{l}\text { SMALLEST attainable DT. Smallest floating point timestep size that is greater than } \\
\text { DTMIN and is determined via Eq (1) }\end{array}$ \\
\hline af & Amplification Factor. Factor that is used to increase the timestep size. \\
\hline rf & Reduction Factor. Factor that is used to reduce the timestep size \\
\hline dtadj & DT ADJustment. \\
\hline curtmi & CURrenT next MInor edit time \\
\hline curtmj & CURrenT next MAjor edit time \\
\hline curtpl & CURrenT next PLot time \\
\hline curtrs & CURrenT next ReStart time \\
\hline endtime & problem END TIME \\
\hline edittime & the next EDIT TIME target (of any kind, major, minor, etc.) \\
\hline dtlimit & DT upper LIMIT based on error mitigation \\
\hline dtsmallest & $\begin{array}{l}\text { SMALLEST attainable DT. The smallest timestep size that is greater than DTMIN and } \\
\text { is determined via Eq (1) }\end{array}$ \\
\hline
\end{tabular}

\subsection{Module Internal Subprograms}

These subroutines were abstracted from R-DTSTEP and/or P-DTSTEP. They were modified to work appropriately for both, such as adding a starting point to subroutine findhalving. In some cases, further improvements were made in resolving user problems, such as adding the creation of qdtsmallest to mkdtsmall. These subroutines were then unneeded in R-DTSTEP and P-DTSTEP and were removed.

Table 12. Integer Timestepping Local Variables in TARGETMOD

\begin{tabular}{|l|l|l|}
\hline calctimehy & real function & $\begin{array}{l}\text { Implements New Algorithm } 3 \text { to calculate the time given an integer } \\
\text { value of time relative to the beginning of the timestep card. }\end{array}$ \\
\hline check8000 & Logical function & Determines if the 8000 message needs to be exchanged. \\
\hline findhalving & Subroutine & $\begin{array}{l}\text { Implements New Algorithm } 9 \text { to find the largest timestep size smaller } \\
\text { than a given the value (provided in the calling sequence) by } \\
\text { successively halving a starting value. The start value may be } \Delta T_{\text {MAX }} \text { or } \\
\text { an unusual timestep size. }\end{array}$ \\
\hline isnormaldt & Logical function & $\begin{array}{l}\text { Applies New Algorithms } 7 \text { and } 8 \text { to determine if the current timestep } \\
\text { size is normal. A timestep is declared to be normal if the floating } \\
\text { point value of the timestep size and the value that is calculated using } \\
\text { Eq (1) differ by no more than } 10^{-10} \text { seconds. }\end{array}$ \\
\hline mkdtsmall & Subroutine & $\begin{array}{l}\text { Applies New Algorithm } 5 \text { to reset the floating point representation of } \\
\text { both the timestep size (dt) and the time (timehy) based on their } \\
\text { integer equivalents for normal timestep sizes. }\end{array}$ \\
\hline movedtdat & $\begin{array}{l}\text { Implements New Algorithms } 1 \text { and } 2 \text { to calculate the variables } \\
\text { dtsmallest and qdtsmallest based on the DTMIN and DTMAX. }\end{array}$ \\
\hline Subroutine & $\begin{array}{l}\text { This subroutine is used to advance or reduce both the floating point } \\
\text { value of time (timehy) and the integer representation of time (irtime). } \\
\text { If the timestep is normal, the code alters the integer representation } \\
\text { of time and then uses the calctimehy function to calculate timehy. If } \\
\text { the timestep size is unusual, the value of timehy is modified and the } \\
\text { integer representation of the new time is calculated using the }\end{array}$ \\
\hline
\end{tabular}




\section{Implementation of a New DTSTEP Algorithm for use in RELAP5-3D and PVMEXEC}

\section{Completion Report}

\begin{tabular}{|l|l|l|}
\hline & & time2int function. \\
\hline time2int & $\begin{array}{l}\text { Integer } \\
\text { function }\end{array}$ & $\begin{array}{l}\text { Applies New Algorithm 4 to calculate the integer representation of a } \\
\text { floating point value of time. The returned value is relative to the } \\
\text { beginning of the timestep card. }\end{array}$ \\
\hline
\end{tabular}

Handling this module during maintenance and installation introduces a new nuance. It is a huge maintenance difficulty to maintain copies of the same file in two directories because eventually one copy or the other is changed without the other. The use of a Unix or Linux link can mitigate the issue; however, links can be broken. It is better to have just one file.

With the decision to retain just one copy of module idtmod, the installation process was affected. The module can reside in either the RELAP or the PVMEXEC directory, but not both. During installation, either linking or copying could provide the other directory with the files it needs. This was implemented.

\subsection{Development and Debugging}

The elements of integer timestepping described in Sections 2, 3, and 4 were developed early, while those in Section 5 were developed during the development. Similarly, all internal subroutines and most new variable names were created during the development process.

Development was performed in small increments because of the requirement to not change answers (except for correcting errors and for slightly improved time tracking). It was found that when numerous conversions were made, the likelihood of altering calculations increased. Therefore, changes were introduced in small groups, sometimes as small as three or four statements. After introducing changes, the complete set of standard installation problems, including PVM problems were rerun and compared against the base code output.

Eventually, the integer timestepping results began to differ slightly from the original due to more accurate time tracking. From this point onward, a second base case was established and output from developmental code was compared against it. This occurred and was repeated several times in the development process. This process is summarized as .

\section{Incremental Development Strategy}

1. Develop means to implement small, independent change

2. Code the few changes

3. Run all test cases

4. Compare results

5. If differences are found, decide if there is an error, or improved accuracy

a. If error, determine source and return to 1.

b. Otherwise, done

\subsection{Development Issues}

The two issues causing the greatest challenge to development were:

1. The problem of finding a suitable coding expression for an integer concept

2. Control coding 


\section{Implementation of a New DTSTEP Algorithm for use in RELAP5-3D and PVMEXEC}

Completion Report

The issue of finding a suitable expression of an integer concept in coding is not new. It happens that coding something one way causes an error in one or more test cases, but that an alternative programming-equivalent set of coding runs correctly. This is sometimes revealed by placing the RELAP5 code on another platform and running the test set, but more often occurs on the developmental platform. The former is a well-known problem caused by differences in compilers, different release levels of the same compiler, math libraries and other operating system differences, and even in PVM interaction with the operating system level. The latter is caused by glitches in the compiler and in PVM interaction with the operating system.

The result is that implementation of the integer concepts was often not as straightforward as it would seem. For example, the ans79.i test problem uses huge timesteps. This caused New Algorithm 1 to fail when 32-bit integers were used, but once this was traced and understood, the problem worked properly when 64-bit integers were used. Other examples include the form of integer if-tests and the updating of time targets.

The second development issue is control coding. In many places, the means of updating timerelated quantities was dependent upon whether RELAP or PVMEXEC was in control, or when PVM was in control, the kind of coupling was in use, particularly synchronous vs. asynchronous. In some places it was necessary to completely separate the control code on these bases, and in others it was not. For clarity, consistency and efficiency of coding though, redundancy is undesirable. Control coding required numerous reworkings for this reason.

Finally, Parallel Virtual Machine (PVM) exhibits different behavior on different platforms. These failures were caused by the inability of the operating system to obtain a PVM daemon when requested, which mostly occurred if PVM jobs were run in succession in a script. This made it appear that PVM test cases were failing when they were not and a rerun would actually work. Optional "permission to terminate" source coding was created to address this and PVM scripts had "post-run" delays and other correction coding added. These devices reduced but did not completely eliminate the problem.

\subsection{Testing and Debugging}

In order to assess the correctness of the integer time-stepping, implementation the existing set of test cases was augmented considerably of the transmittal set. An initial problem, time8, with 8 timecards that tested different aspects of timecard handling by RELAP5-3D run under PVMEXEC control was the first addition. Input files associated with User Trouble Reports (UTR) were also added; this included some problems that mimicked actual UTR when the original input files were unavailable. Some special cases, designed by Dr. Walter Weaver, that create and test failure handling in coupled problems were also incorporated. Finally, a huge test matrix that tests every kind of coupling and many other aspects of coupled calculations was constructed.

The so-called Weaver test set is presented in Section 8.2.1, the tests related to UTE in 8.2.2, and the so-called DTSTEP Test Matrix comprises Section 9. 


\title{
Implementation of a New DTSTEP Algorithm for use in RELAP5-3D and PVMEXEC
}

\author{
Completion Report
}

\subsection{Debugging: The Weaver test set}

The so-called Weaver test set consists of 9 test cases designed to check various combinations of the DTSTEP time-step repeat logic and the ability of the code to detect errors and shut down without creating a hung machine condition.

Code modifications were necessary in subroutines VFINL and TRAN. The coding necessary for these tests is marked with pre-compiler directives testpvm1 through testpvm9. The entire RELAP5-3D code must be recompiled separately for each test case. This is automated in the controlling Linux script named RUNW.

No errors were found in DTSTEP as a result of these tests.

\subsection{The TestDt Tests}

The next group of tests had two components: available DTSTEP UTR input and the attempted recreations of unavailable UTR input. These input models are located in the run/TestDt directory of the RELAP5-3D source distribution. The Linux script created to run the test set is named runv_dt. The problems are listed in Table 11.

Note that files in Table 11 with the "ii" extension are the name of the PVMEXEC master input file; there are matched input files for the RELAP5-3D processes that the executive spawns. These input file names start the same but differ on the last letter; their extension is "i." For example, time8.ii spawns a process whose input file is time 8 p.i.

All available models in Table 11 ran with version R3D244B. Although input pvmcore6_20x.ii, unusual.i, and r5_melcorx.ii helped pinpoint errors in the implementation and eventually ran, other recreations failed to reproduce the reported behavior after repeated modifications and were thus unsuccessful in advancing the debugging and testing process.

The pvmcore6_20x.ii problem has 6 connection between master and slave in a semi-implicit coupling was of interest for both the impact of FORTRAN 95 conversion, a simultaneous project that was in its final stages, and for the ability of the BPLU solver to handle that many connections. It was used to help solve a RELAP5-3D/MELCOR coupling error wherein the restart at 10 seconds did not output the same values that the continuous run to 20 seconds had.

Table 13. TestDt input models for debugging unavailable input models

\begin{tabular}{|l|l|}
\hline \multicolumn{1}{|l|}{ Name } & Description \\
\hline ed_UP04028.i 1 & Available/Extended input models \\
\hline vhtr03009.i & Input model for User Problem 04028 \\
\hline time8a.i i & Input model for User Problem 03009 \\
\hline time8.ii & A fast-running version of time8.i \\
\hline unusual.i & The original 8-timecard input model described in Sec. 8.2 \\
\hline \multicolumn{1}{|c|}{ Section 2 } & A fast-running problem with a 4 different challenging unusual time issues \\
\hline r5_melcorex.ii & $\begin{array}{l}\text { Attempt to recreate the RELAP5-MELCOR problems reported in July } 2009 \\
\text { Slave process input: r5_melcoreb1.i, and r5_melcoreb2.i }\end{array}$ \\
\hline r5_cobra1109x.i & Attempt to recreate a RELAP5-3D coupled to COBRA calculation that hangs at 1107 \\
\hline
\end{tabular}




\section{Implementation of a New DTSTEP Algorithm for use in RELAP5-3D and PVMEXEC}

\section{Completion Report}

\begin{tabular}{|l|l|}
\hline & sec \\
\hline Restart2ndx.ii & $\begin{array}{l}\text { Attempt to recreate the problem with a restart of a restart of a coupled calculation } \\
\text { from an unavailable UTR }\end{array}$ \\
\hline $\begin{array}{l}\text { pvmcore6x_20.ii } \\
\text { pvmcore6x.ii } \\
\text { pvmcore6xr.ii }\end{array}$ & $\begin{array}{l}\text { Semi-implicit coupling input model with } 6 \text { coupling junctions. The "6x" file runs from } \\
\text { runs from } 0 \text { to } 20 .\end{array}$ \\
\hline
\end{tabular}

The input set comprised of r5_melcorex.ii, r5_melcoreb1.i, and r5_melcoreb2.i was useful in identifying an error in the coupling of RELAP5-3D to MELCOR involving a non-positive timestep.

Unusual.i was created to severely test the unusual timestep handler, New Algorithm 6, and it revealed some problems that were also corrected.

\subsection{The Proprietary Tests}

In order to provide an algorithm with the required level of robustness, a comprehensive test program was required. This test program consisted of a suite of proprietary problems that either expressed algorithmic failures in previous versions of the algorithm or problems that had been designed to test specific features in the algorithm. During the development process, new failure mechanisms in the algorithm were discovered, these proprietary problems were then added to the test suite. These problems are not available for distribution but played a fundamental role in debugging RELAP5-3D.

Every version of the algorithm that was developed was tested against the entire test suite, as it existed at the time. A checklist was developed and used for each test version. A sample checklist is included as Table 1. The checklist recorded the compilation date and time for both the RELAP5-3D and PVMEXEC executable. This information was recorded to permit reproducibility of the results, if desired.

Another unique aspect of this development effort was that every version of the files containing the shared module idtmod and the dtstep subroutine, for both RELAP5-3D and PVMEXEC programs, were automatically saved in a repository immediately following the creation of executables. This process made it possible to recreate the source code that was used for any test version of the code and provided the ability to quickly identify the changes that either solved or created problems with the algorithm.

The final version of the test suite included several smaller test suites which included:

- The DTSTEP Test Matrix (Section 10)

- The proprietary installation problems (verify)

- A test suite of 204 different problems executing RELAP5-3D as a standalone program.

- The Weaver Failure Tests (Sec. 9.3)

- A suite of restart problems that couple RELAP5-3D and COBRA-IE (cobra_restart) This is a suite of problems that is used to make sure that coupled problems can be restarted during the solution process without altering the solution. This test suite 


\section{Implementation of a New DTSTEP Algorithm for use in RELAP5-3D and PVMEXEC}

Completion Report

consists of a base case and then 5 different restart cases. Each of the restart cases starts from the previous restart case and the results are compared to ensure that results are the same to machine precision.

- A collection of 5 different User problems (User1 - User5) These problems represent specific problems that were uncovered in the algorithm at various points in the development process. These problems were long running jobs, with the longest taking over 4 days to reach the point of failure.

A checklist for running this collection of test suites was developed. An example is shown in Table 20 in Appendix A.

\subsection{The DTSTEP Test Matrix}

The DTSTEP Test Matrix was was designed as a huge collection of test cases that would exercise every set of important conditions and relevant paths through RELAP5-3D DTSTEP (R-DTSTEP) for coupled problems. Developed simultaneously with the debugging methods of Sections 8.2 through 8.4, it was designed to systematically test each mode of coupling on all known important failure mechanisms. The design is given in Sec. 9.1. The 199 debug card design is in Sec 9.2. Subroutines created to implement it are given in Sec. 9.3. The modifications to existing subroutines in reported in Sec 9.4. The large collection of scripts that run it are described in Sec. 9.5. The input models, including the newly created ones, are described in Sec 9.6. The debugging effort is reported in Sec. 9.7.

\subsection{Test Matrix Design}

The Test Matrix is comprised of 2856 separate test cases, each testing a combination of logic paths through the code that have generated PVM coupling errors. The test matrix is divided into five packages of test cases; each addresses a different aspect of the DTSTEP. Each contains two or more copies of the next smaller package applied differently. The packages are shown in Table 12 along with the number of separate tests in each package.

Table 14. Test Matrix levels of testing DTSTEP

\begin{tabular}{|l|l|r|}
\hline Name & Description & \# tests \\
\hline Basic & Test special timestepping conditions (halving/doubling/repeating) and failures & 17 \\
\hline Package A & Vary the kind of timestep size with Basic Group & 34 \\
\hline Package B & Vary Time Target when it is applied on Group A & 102 \\
\hline Package C & Vary type of time target: Normal, unusual, sequence, every step on Group B & 408 \\
\hline Package D & The 7 PVM coupling modes on Group C & 2856 \\
\hline
\end{tabular}

An example would be a test that repeats the timestep (Basic) when dt is minimal (package A) at a plot pt. (package $B$ ) that is an unusual time (package $C$ ) in semi-implicit coupling (package $D$ ). 


\section{Implementation of a New DTSTEP Algorithm for use in RELAP5-3D and PVMEXEC}

Completion Report

The Basic Set is comprised of simple conditions that are known to have caused failures in existing PVM-coupled input models. There are single condition tests (designated a1 through e3) and tests comprised of combinations of single conditions. These are summarized in Table 13.

Table 15. The 17 Basic Tests of the DTSTEP Test Matrix

\begin{tabular}{|l|c|l|}
\hline $\begin{array}{l}\text { Test } \\
\text { Designation }\end{array}$ & $\begin{array}{l}\text { Test } \\
\text { Number }\end{array}$ & Description \\
\hline a. Repeat Only (Note: These all travel the same logic path through RELAP5-3D DTSTEP.) \\
\hline a1. & 1 & succes=5 (Air appearance, velocity flip-flop, water-packing) \\
\hline b. Reduction Only (halving) \\
\hline b1. & 2 & Exceed Material Courant Limit (MCL) \\
\hline b2. & 3 & PVM transmits reduced step \\
\hline c. Repeat with Reduction \\
\hline c1. & 4 & succes=1, Excessive Mass error \\
\hline c2. & 5 & succes=2, Thermodynamic property failure \\
\hline d. Amplification (doubling) \\
\hline d1. & 6 & MCL and mass error check \\
\hline e. Code Termination for FAlLURE condition \\
\hline e1. & 7 & fail=-.true. \\
\hline e2. & 8 & Variable volume minimum size (succes=6) \\
\hline e3. & 9 & Executive: pvmerr /= 0 \\
\hline Combinations & & Conditions occurring on successive steps \\
\hline a1-b1 & 10 & a1 then b1 \\
\hline a1-b2 & 11 & a1 then b2 \\
\hline a1-c1 & 12 & a1 then c1 \\
\hline a1-c2 & 13 & a1 then c2 \\
\hline a1-b1-d1 & 14 & a1 then b1 then d1 \\
\hline a1-b2-d1 & 15 & a1 then b2 then d1 \\
\hline a1-c1- d1 & 16 & a1 then c1 then d1 \\
\hline a1-c2- d1 & 17 & a1 then c2 then d1 \\
\hline
\end{tabular}

\subsubsection{Package A Timestep Sizes (34 tests)}

Package $A$ applies the Basic Set twice, once with a normal sized step, and once with $\Delta t_{\text {small }}$. The two instances of the Basic set are named $A 1$ and $A 2$. Thus,

- A1 Basic set (9) and 8 Combinations at non-minimal time step

- A2 Basic set (9) and 8 Combinations at minimal time step

\subsubsection{Package B Time Targets (102 tests)}

Package $B$ applies package $A$ thrice, once at a minor edit, then at an explicit exchange time, and at the end of transient. The instances of Package A are named B1, B2 and B3. Thus,

- B1 Package A tested at/near minor edit time (represents plot/major/restart edits)

- B2 (where applicable) Package A tested at/near message exchange time

- B3 Package A tested at/near Transient end 


\section{Implementation of a New DTSTEP Algorithm for use in RELAP5-3D and PVMEXEC}

Completion Report

\subsubsection{Package C Normal/Unusual Time}

(408 tests)

Package $C$ applies package $B$ four times, once at multiple of DTMAX, then at an unusual time, at a sequence of normal and unusual targets, and at every timestep. The instances of Package $B$ are named $\mathrm{C} 1, \mathrm{C} 2, \mathrm{C} 3$ and $\mathrm{C} 4$. Thus,

- C1 Package B: Time targets are integer multiples of DTMAX

- C2 Package B: Time targets are unusual (non-integer multiples of DTMAX)

- C3 Package B: normal target, followed by unusual, followed by normal

- C4 Package B: Targets specified at every step via RELAP5-3D or Executive.

\subsubsection{Package D Coupling Configurations (2856 tests)}

Package $D$ applies package $C$ seven times, as indicated in the bullets. The instances of Package $C$ are numbered D1, through D7. Thus,

- D1

Stand alone RELAP5-3D

- D2

Explicit Asynchronous coupling RELAP5-3D/Relap5-3D

- D3

Semi-implicit Synchronous coupling RELAP5-3D/Relap5-3D

- D4

Simultaneous coupling (Explicit Asynchronous and Semi-implicit Synchronous) RELAP5-3D/Relap5-3D/RELAP5-3D

- D5

Explicit Synchronous coupling RELAP5-3D/Relap5-3D

- D6

Explicit Asynchronous coupling RELAP5-3D/Relap5-3D with 1st as leader

- D7

Synchronous single RELAP5-3D controlled by Executive

Note that there are somewhat fewer tests than the upper limit of 2856 tests in the matrix. For instance, asynchronous coupling implies no testing of Basic Test b2 and that there can be no testing of sub-package B2 with semi-implicit coupling, D4. There are other combinations that make no sense to test as well. The run scripts prevent non-sensical cases from being run; see Sec. 9.4.

The designation of a single case within the DTSTEP Test Matrix is unique. It is named according to its groupings, from largest package to smallest. The format of every case is:

Dv CW Bx Ay CASE z

An example is D2 C4 B3 A2 CASE 10 indicates that the case tests asynchronous coupling with minor edits output every time step wherein air-appearance followed by a violation of the material Courant limit occurs just at the end of the transient. The subroutines described in Sec. 9.3-9.5 use no spaces in the naming the cases. E.G. DvCwBxAyCASEz

The test matrix is designed to take a single input model, one from each coupling configuration in Package $D$, and modify it to apply the specifications of the other packages to the input deck to create an altered deck that exactly defines the test to RELAP5-3D. Modifications to the input deck are made by scripts and include the addition of the new 199 debug card; the subroutines convert the input into logic flowpath control.

\subsection{The 199 debug card}

Part of the Test Matrix design is a new input card, number 199, contains information about which subroutine to test, what to test, and when to test it. However, the 199 card is designed to be a generic system for creating user-controlled debug information for use ANYWHERE in RELAP5-3D. 


\section{Implementation of a New DTSTEP Algorithm for use in RELAP5-3D and PVMEXEC}

Completion Report

The Generic 199 card is a standard RELAP5-3D input card. Its format is:

199 Word1 Word2 Word3 Word 4

- Word 1 A character string that names a subroutine (group) to debug

- Word 2 A keyword that indicates what aspect to debug

- Word 3 A number indicating the advancement to activate the debugging

- Word 4 A number indicating the advancement to terminate the debugging

The 199 card is limited to DTSTEP debugging currently. Therefore, Word 1 must be DTSTEP and Word 2 must be a basic-case-number, $z$, followed by package A subset indicator, either A1 or A2. Thus the 199 card Word 2 must have the form zAy. The 199 format is:

$$
199 \text { DTSTEP "zAy" T1 T2 }
$$

where $1 \leq z \leq 17,1 \leq y \leq 2$, and the test activates on advancement $\mathrm{T} 1$ and terminates on $\mathrm{T} 2$. By choosing $\mathrm{T} 1$ and $\mathrm{T} 2$ properly, the time-target aspect controlled by package $\mathrm{B}$ is incorporated into the test case.

Thus, the 199 card creates the aspects of the test case controlled by packages B, A, and Basic, but not packages $C$ and $D$; those are controlled by the scripts. Exercising the specified Test Matrix case is left to the new Test Matrix subroutines.

\subsection{The new Test Matrix subroutines}

Two subroutines, with 4 internal subroutines, were created to implement the DTSTEP Test Matrix; they are described in Table 14. Module Testmod carries the data created by the input one to the transient routine. Testmod is described in Sec 6.4.4 in Tables 6 and 6a.

Table 16. Subroutines created to implement the DTSTEP Test Matrix

\begin{tabular}{|l|l|}
\hline Major Subroutine & Description \\
\hline rdebug & $\begin{array}{l}\text { Read user-control debug data from } 199 \text { card, check it and print diagnostics for for } \\
\text { incorrect form or data. Interpret data as Table 13 “Test Designation" (with no } \\
\text { dashes) in Testmod variable ctest. }\end{array}$ \\
\hline testDtstep & $\begin{array}{l}\text { Create tests of various logic paths through DTSTEP for the DTSTEP Test Matrix by } \\
\text { interpreting ctest and setting variables cpurei(4:5), done, errmax, fail, success, } \\
\text { and/or iTestDtstep appropriately on the user-designated timesteps only. }\end{array}$ \\
\hline Internal Subroutine & Within testDtstep \\
\hline basicSet & Sets DTSTEP variables to implement test from Table 13 “Test Designation" \\
\hline combine1_4 & Sets DTSTEP variables to implement test of Table 13 first 4 combinations. \\
\hline combine5_8 & Sets DTSTEP variables to implement test of Table 13 2 4 combinations. \\
\hline packageA_set & Conditionally calls subroutines basicSet, combine1_4, or combine5_8. \\
\hline
\end{tabular}

The new subroutines are internally documented. Except for a few local variables, every variable used by them is listed in the preceding Tables of variables. 


\title{
Implementation of a New DTSTEP Algorithm for use in RELAP5-3D and PVMEXEC
}

\author{
Completion Report
}

\subsection{Updates in support of the Test Matrix}

Subroutine RNEWP was adjusted to call RDEBUG. Two RELAP5-3D subroutines TRAN and DTSTEP were modified to implement the Test Matrix in the transient. Both access the data in Testmod to activate the tests. Three more subroutines were modified after version $\mathrm{r} 3 \mathrm{~d} 244 \mathrm{~b}$ to identify lapses in writing major/minor/restart edits in Test Matrix output files automatically. A protocol to reduce PVM daemon acquisition errors was installed in PVMEXEC TRAN and RELAP5 TRNCTL.

In RELAP5-3D TRAN, the Test Matrix is activated by a call to testDtstep just above the coding for the first Weaver Test, marked testpvm1; see Sec. 8.3 above. It is called again just prior to the call to DTSTEP. Both calls occur in coding marked with pre-compiler directive pvmcoupl so that it can be removed for most client groups.

In RELAP5-3D DTSTEP, the coding is implemented with if-tests on variable iTestDtstep. All these if-tests are also marked with pre-compiler directive pvmcoupl. The implementation in DTSTEP is described in Table 15.

Table 17. Implementation of Test Matrix basic tests within RELAP5 DTSTEP

\begin{tabular}{|l|l|l|}
\hline iTestDtstep & Description & Implementation in RELAP5-3D DTSTEP \\
\hline 1 & Exceed Courant limit & $\begin{array}{l}\text { Forced by setting } \mathrm{dtx} \text { to the larger of } \Delta \mathrm{t}_{\text {small }} \text { and } 0.9 \mathrm{x} \Delta \mathrm{t}_{\mathrm{i}+1} \text { in Sec. } \\
10.3 \text { of DTSTEP }\end{array}$ \\
\hline 2 & Halving dt & $\begin{array}{l}\text { Forced by resetting } \mathrm{dt}=\mathrm{dt} / 2 \text { in DTSTEP Sec } 11.1 \text { (synchronous } \\
\text { coupling only) just before the proposed } \mathrm{dt} \text { is sent to PVMEXEC. } \\
\text { This is necessary so that PVMEXEC has same dt. }\end{array}$ \\
\hline 3 & PVM error condition & $\begin{array}{l}\text { Forced by setting pvmerr=1 in four places: in initialization, } \\
\text { within IntTarg8000, in Sections } 2.3 \text { and 7.1 of DTSTEP. This is } \\
\text { necessary to avoid creating a machine hang. }\end{array}$ \\
\hline 4 & Minimal step size & Forced by setting dtx to twice dtmin in Sec 10.3 of DTSTEP \\
\hline
\end{tabular}

In order to verify that the major, minor, and restart edits are occurring as they should, coding was added to count the number of each, and to output those values with each message about the appropriate edits in the "printed output" file. These changes occur in MAJOUT, MIREC, and RSTREC. Even then, the scripts automate the process of checking the values for correctness.

A common problem related to running numerous successive PVM jobs was failure to obtain a PVM daemon to start running a job. This would occur when the Test Matrix was run. To overcome this, an optional "permission to terminate" (also called "Mother may I") protocol with PVM was developed. The message tag is 8100.

Just before the return statement in TRNCTL of RELAP5-3D, an 8100 message is sent to the Executive. This is followed by a PVM-Receive statement. The process cannot terminate until either an 8100 message from PVMEXEC TRAN arrives, or the wait time is exceeded. The executive has a receive loop similar to an 8005 message tag in TRAN and only after all processes have requested termination may the executive broadcast the terminate message to all. If one of the child processes fails, the PVM Executive wait time will be exceeded and the normal error message will be written from the main program. 


\title{
Implementation of a New DTSTEP Algorithm for use in RELAP5-3D and PVMEXEC
}

\author{
Completion Report
}

\subsection{The New Scripts}

A large set of Linux scripts was created to organize, set-up, run, and check the DTSTEP Test Matrix. These are summarized in Table 16.

Table 18. The new scripts that operate the DTSTEP Test Matrix

\begin{tabular}{|c|c|c|c|}
\hline Script Name & Function & Calls & Called by \\
\hline base17 & $\begin{array}{l}\text { Run } 17 \text { basic test cases of DTSTEP in sequence. } \\
\text { Its } 4 \text { call arguments describe the choice of D-coupling, C- } \\
\text { kind, B-target, and A-dt-size }\end{array}$ & $\begin{array}{l}\text { mk199card } \\
\text { settimecards } \\
\text { testsuccess }\end{array}$ & packageA \\
\hline BasicCase & $\begin{array}{l}\text { The centerpiece of the scripting system. Given the } 5 \\
\text { numbers that describe a test, it selects the input model } \\
\text { template and prepares the executive input file, makes } \\
\text { copies of the slave process input files, adding a debug card, } \\
\text { and modifying their time cards. It also runs the code, } \\
\text { examines the output and determines if it is satisfactory } \\
\text { before cleaning up and renaming the output for later } \\
\text { manual examination. BasicCase can be run manually for } \\
\text { any of the test matrix's } 2856 \text { cases. }\end{array}$ & $\begin{array}{l}\text { checkCase } \\
\text { mk199card } \\
\text { settimecards } \\
\text { testsuccess } \\
\text { pvmexec.x } \\
\text { relap5.x }\end{array}$ & base17 \\
\hline CheckCase & $\begin{array}{l}\text { Determine if a case makes sense to run. Return with status } \\
\text { flag } 1 \text { if the Basic Case makes no sense to run. Also display } \\
\text { a message explaining why. }\end{array}$ & none & BasicCase \\
\hline editCount & $\begin{array}{l}\text { Counts the number of restart, major, and minor edits in } \\
\text { the RELAP5-3D output file. }\end{array}$ & None & BasicCase \\
\hline mk199card & $\begin{array}{l}\text { Creates the } 199 \text { debug card to append to the end of a } \\
\text { RELAP5-3D input deck that implements the test specified } \\
\text { by packages B, A and BasicCase (time, dt-size and basic } \\
\text { case number) }\end{array}$ & & BasicCase \\
\hline packageD & $\begin{array}{l}\text { Run all } 2856 \text { problems of the DTSTEP Test Matrix by calling } \\
\text { packageC for all } 7 \text { types of coupling tests. Stores the } \\
\text { results in a log file. }\end{array}$ & packageC & runTest \\
\hline packageC & $\begin{array}{l}\text { Runs } 408 \text { test cases by calling packageB for } 4 \text { kinds of time } \\
\text { targets (normal, unusual, combo, every-step). }\end{array}$ & packageB & packageD \\
\hline packageB & $\begin{array}{l}\text { Runs } 102 \text { test cases by calling packageA for } 3 \text { target types } \\
\text { (plot, exchange, transient-end) }\end{array}$ & packageA & packageC \\
\hline packageA & $\begin{array}{l}\text { Run the } 34 \text { basic test cases for the conditions of } A 1 \text { and } A 2 \\
\text { for a normal and smallest time step respectively. }\end{array}$ & base17 & packageB \\
\hline rerun & $\begin{array}{l}\text { Finds and reruns all Test Matrix cases that ran and } \\
\text { produced the result "Unsatisfactory." }\end{array}$ & $\begin{array}{l}\text { Creates } \\
\text { reRunTest }\end{array}$ & none \\
\hline runTest & $\begin{array}{l}\text { Set up the DTSTEP Test Matrix, making certain that the } \\
\text { RELAP5-3D, PVMEXEC, and pvmcatchout executables and } \\
\text { all the fluid property files are accessible. Also deletes all } \\
\text { output files from any previous Test Matrix run in the } \\
\text { directory. }\end{array}$ & packageD &..$/$ runT \\
\hline..$/$ runT & $\begin{array}{l}\text { Runs the DTSTEP Test Matrix and DTSTEP Extra problems. } \\
\text { Checks first that the code was installed with PVM on. } \\
\text { The log file defaults to the date followed by_TM. }\end{array}$ & $\begin{array}{l}\text { runTest } \\
\text { (Test Matrix) } \\
\text { runv_dt } \\
\text { (of Sec. 8.4) }\end{array}$ & None \\
\hline seek & $\begin{array}{l}\text { Checks that a single success value occurred in at least once } \\
\text { in a group of files. }\end{array}$ & None & testsuccess \\
\hline
\end{tabular}




\section{Implementation of a New DTSTEP Algorithm for use in RELAP5-3D and PVMEXEC}

\section{Completion Report}

\begin{tabular}{|l|l|l|l|}
\hline settimecards & $\begin{array}{l}\text { Creates timecards that implement the test called for by } \\
\text { packages B, C, and D. }\end{array}$ & None & BasicCase \\
\hline testsuccess & $\begin{array}{l}\text { Compares the output of a run of the PVM-Executive (or } \\
\text { RELAP5-3D in the case of set D1) against the expected final } \\
\text { message. }\end{array}$ & seek & BasicCase \\
\hline
\end{tabular}

All scripts in Table 16 are located in the TestMatrixDt subdirectory of the "run" directory, except runT which is located in "run." More complete testing is available by running runT from the "run" directory of RELAP5-3D; this will run all test cases listed in Sec. 8.4 and the Test Matrix.

As described in an earlier Section, the 199 card does not communicate the D- and C-level aspects of a Test Matrix test case. These are introduced by two mechanisms. Script settimecards of Table 15 modifies the timecards of an input deck to implement the C-level control. BasicCase selects the input deck that corresponds to the D-level coupling of the specific test case.

\subsection{The Input Models}

Simple input models are used as the basis for all tests of a particular kind of D-level coupling. The input models are given in Table 17.

Table 199. Test Matrix base input tests, the models and their sets of input files

\begin{tabular}{|c|c|c|c|}
\hline D-level & $\begin{array}{l}\text { Base Input } \\
\text { File }\end{array}$ & $\begin{array}{l}\text { Other input files of } \\
\text { base set }\end{array}$ & Description \\
\hline 1 & edhtrk.i & & $\begin{array}{l}\text { RELAP5-3D standalone. Edward's pipe model (with } \\
\text { extras). Reference [c]. }\end{array}$ \\
\hline 2 & pvmedax.ii & $\begin{array}{l}\text { pvmedac.i - child } \\
\text { pvmedap.i - parent }\end{array}$ & $\begin{array}{l}\text { Asynchronous explicit coupling test case. } \\
\text { Edward's pipe }\end{array}$ \\
\hline 3 & pvmcore & $\begin{array}{l}\text { pvmcorep. } \mathrm{i} \text { - primary } \\
\text { pvmcorec. } \mathrm{i} \text { - core }\end{array}$ & $\begin{array}{l}\text { Semi-implicit (synchronous) coupling } \\
\text { Christensen model }\end{array}$ \\
\hline 4 & pvm3way & $\begin{array}{l}\text { pvm3wayp.i - primary } \\
\text { pvm3wayc.i - core } \\
\text { pvm3wayb.i - bypass }\end{array}$ & $\begin{array}{l}\text { Semi-implicit (primary to core) AND explicit } \\
\text { asynchronous (primary to bypass) coupling } \\
\text { Christensen model. Reference [d]. }\end{array}$ \\
\hline 5 & pvmedspx.ii & $\begin{array}{l}\text { pvmedsc. } \mathrm{i} \text { - child } \\
\text { pvmedsp.i - parent }\end{array}$ & $\begin{array}{l}\text { Synchronous explicit coupling } \\
\text { Edward's pipe }\end{array}$ \\
\hline 6 & pvmeds10x.ii & $\begin{array}{l}\text { pvmeds10f.i - follower } \\
\text { pvmeds10l.i - leader }\end{array}$ & $\begin{array}{l}\text { Asynchronous explicit conserving } \\
\text { Edward's pipe }\end{array}$ \\
\hline 7 & pvmvesselx.i & pvmvesselp.i & $\begin{array}{l}\text { Executive oversees single RELAP5-3D running a full } \\
\text { Christensen vessel model }\end{array}$ \\
\hline
\end{tabular}

There are only two basic input models, one based on the Edward's blowdown test, and the other based on the Christensen vessel model. These problems are simple and fast running as compared with, for example, a full plant model or the standard Typical PWR model that has serious modeling deficiencies that cause many code failures. There are two reasons for this.

The first reason is time; there are 2856 test cases and the time to run the full Test Matrix, at the time, was 2.5 to 4 hours, depending on the computer platform. Changing from Edward's pipe that runs in a few seconds to a problem that runs in minutes or longer would make the Test Matrix too slow for debugging purposes. Increasing the runtime of the base input models by 


\section{Implementation of a New DTSTEP Algorithm for use in RELAP5-3D and PVMEXEC

using something that took a minute to run would increase runtime by a factor of 20 or more and increase Test Matrix runtime from 4 hours to 4 days. This was unacceptable as the test matrix was run hundreds of times during the course of testing and debugging.

The second reason is failures. The PVM system would fail by itself for various reasons including inability to get a PVM daemon, required failures (basic cases 7, 8, and 9), and DTSTEP bugs. Simple, stable models were needed to identify these errors. Errors in other parts of the code that may or may not have originated from coding bugs in one of the DTSTEP programs would only complicate and further lengthen the overall debugging process.

\subsubsection{The New Input Model}

To perform coupling tests in the D4 category, a new simple test problem was required. This was made by decomposing the "pvmcore" problem so that a portion of the core was connected to the primary part of the vessel (including the rest of the core) semi-implicitly, while the bypass was connected to the primary explicitly.

The new problem is called "pvm3way" and a nodding diagram is given in Figure 5.

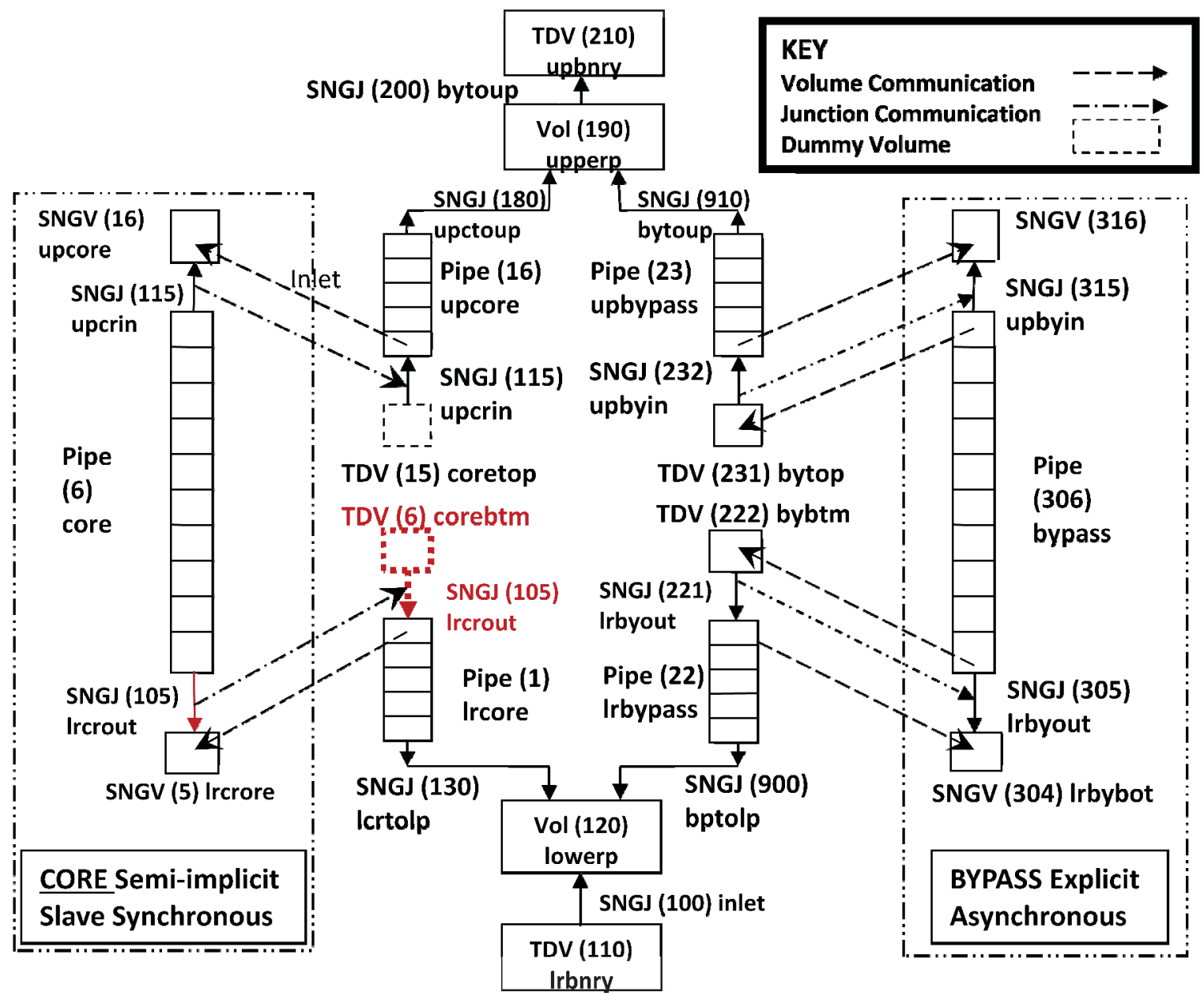

Figure 5. PVM3WAY Nodalization Diagram. 


\section{Implementation of a New DTSTEP Algorithm for use in RELAP5-3D and PVMEXEC \\ Completion Report}

\subsection{Supporting Changes in Existing Coding}

This Section provides a brief summary of the existing subroutines in both RELKAP5-3D and PVMEXEC that were modified to implement the integer timestep algorithm and in support of debugging the coding.

Subroutine pvmfxrec within RELAP5-3D was changed to activate the debug print statements based on the variable from the shared module. The debug print statements now are sent to a common file unit. The unit number is stored in idtmod.

Subroutine trnctl of RELAP5-3D was modified to incorporate the "Permission-to-Terminate" logic for that mitigates PVM failures due to the Operating System shutting down buffers of terminating processes before its data exits to coupled receiving process (Sec. 10.3 and 11.4).

Subroutines majout, miedit, and rstrec were modified to implement the DTSTEP Test Matrix (Sec. 11). Subroutine majout was also modified to display in excess of 14 digits and/or display in hexadecimal for some important debugging quantities.

Module contrl added a new variable, old_pvm_samedt, has been added for the detection of potential infinite loops (Section 5.6 and 12.0).

Subroutine tran of PVMEXEC was modified to incorporate logic for the detection and mitigation of potential infinite loops (Section 5.6). This is done by saving a new variable, old_pvm_samedt, which saves the state of the variable pvm_samedt from the previous timestep. If both pvm_samedt and old_pvm_pvm_samedt are non-zero, the code enforces a timestep size reduction.

Subroutine inputd of PVMEXEC has been modified to provide the ability to provide debug print statements.

\subsection{PVM-coupling and DTSTEP Problems solved}

The following are a list of some of the problems fixed during the development and debugging of the PVM coupling issues with DTSTEP. In this section, R-DTSTEP refers to RELAP5-3D DTSTEP and P-DTSTEP refers to PVMEXEC DTSTEP.

Errors reported here are confined to DTSTEP errors that existed prior to the implement of the integer timestepping algorithm and its development. Many other errors were corrected along the way including errors in the integer timestepping implementation itself, errors in the implementation of the DTSTEP Test Matrix, and errors in other subroutines than DTSTEP.

PVM0901 Code hangs at an unusual endtime Floating point error Description: Hang with floating point time control at unusual times sometimes. Analysis: The user-supplied problem, time8.i, illustrates several types of code failures when RELAP5-3D is run under the control of the PVMEXEC without being coupled to anything. 


\section{Implementation of a New DTSTEP Algorithm for use in RELAP5-3D and PVMEXEC}

Completion Report

Timecard 6 ends at $25200.0021 \mathrm{~s}$ with DTMAX $=0.004$; thus the endtime is unusual and was illustrative of code hangs experienced prior to the integer timestepping project.

Solution: This was resolved by converting to integer timestepping.

PVM0902 Modification for Explosive Doubling PVM conceptual design error Description: During the final timecard, the timestep begins to double, exceeds DTMAX, and continues doubling until the end time is reached.

Analysis: This is a problem in explicit asynchronous coupling only, since for any synchronous coupling the RELAP5-3D timecards have no control and for RELAP5-3D standalone, there are no overriding timecards. An error with synchronous processing arose because the method implemented to overcome explosive doubling affected the synchronous case.

Solution: Adding a clause to prevent synchronous processes from entering the explosive doubling control coding (reported in February 2009) resolved the problem.

PVM0903 Cumulative time in message $8000 \quad$ PVM conceptual design problem Description: The code hangs at the end of timecard; see Section 5.5.

Solution: The cumulative time was added as a ninth data item to both P- and R-DTSTEP. This allows all slave processes to stay in lock-step with the PVM Executive.

PVM0904 Penultimate timecard stop $\quad$ Old DTSTEP conceptual design error UP 03024

Description: When running a D4 Test Matrix case, it was discovered that some asynchronous processes would stop at the end of the second to last timecard.

Analysis and solution: This was tracked down to an error in resetting variable curclm in Section 7.1.1 of R-DTSTEP. This error has been fixed.

\section{PVM0905 Hang when HYDRO sets FAIL PVM conceptual design error}

With D5, code hangs for case D5C2B1A1 case 7. Reported on 24-Mar-09.

Analysis: The algorithm designer did not realize that HYDRO could set FAIL to TRUE. When this happens, RELAP5-TRAN send the (A-OK) 10000 message to the Executive, but later during the time-step DTSTEP correctly begins the shutdown process. Thus both codes arrive at different message wait locations.

Solution: When fail is true from hydro, in the immediately-following PVM coding section, set PVMSUCCESS $=5$ to signal a repeat (because RELAP5 will repeat, then shut down).

PVM0906 Hang in explicit asynch. sequential PVM conceptual design error The code hangs for case D6C1B1A1 cases 10-12. Reported on 25-Mar-09.

Analysis: When the leader of a sequentially coupled problem reaches the end of the interval, it sends a message to the follower to proceed. Normally the follower receives the message in RELAP-TRAN and proceeds through advancements to the rendezvous time. However, if on the same advancement as the receipt of the go-ahead message is received, the code must repeat the advancement, it can repeat the arrival at the receive message. This occurs on, for example, D6C1B1A1 case 10.

Solution: The solution was to create a logical variable that DTSTEP sets whenever the code must repeat the time step. RELAP-TRAN was then modified to access the section of code containing the above-mentioned receive message only when that variable is FALSE. A temporary module, 


\section{Implementation of a New DTSTEP Algorithm for use in RELAP5-3D and PVMEXEC}

Completion Report

BACKMOD, was created to carry it. This was done to make it more obvious when porting to F90 where the variable will be absorbed into a more appropriate module.

PVM0907 Code hang on D4C2B1A1 case 1. PVM design error

Description: Hang with D4C2B1A1 case 1 by stretching past explicit exchange time Analysis: The value of CURTEX was not set to the endtime of the timecard, an unusual time, but rather the multiple of DTMAX just before it. It did not perform the stretch calculation.

Solution: The calculation of integer time targets was modified to incorporate the stretch logic.

Thus if I_CURTEX + 10\%IDTMAX > IDURATION (relative integer time end-of-timecard), then reset I_CURTEX to the nearest multiplier of DTSMALLEST to the end time of the timecard. Then

CURTEX is set to the endtime if I_CURTEX is close to the integer timecard end time, IDURATION.

PVM0908 D4C4B1A2 cases 12 and 16 hung Old DTSTEP error

Description: Hang from repeat condition at minimum dt (expl asyn parallel)

Analysis: When SUCCES $==5$ the code errs at minimal time-steps. In R-DTSTEP Sec. 2.3.3, the code halves DT when SUCCES / 5 despite DT = DT_MIN. It then doubles the time-step in Section 2.4 , which can only be reached when SUCCES $/=1$. When DT $==$ DT_MIN and SUCCES $==5$, the code therefore sets DT $=2 *$ DT_MIN. This is incorrect and causes the slave process to take twice as big a time-step as the executive takes. As a result, they arrive at different messages to send and listen.

Solution: Change the test in DTSTEP Sec 2.3.3 so that both SUCCES /= 5 and DT /= DT_MIN, in order to halve DT. Remove the doubling of DT in DTSTEP Section 2.4. All other paths through the code work the same because the halving and doubling cancel. This change actually makes the code more efficient by reducing wasteful operations.

PVM0909 PVM-dtstep Dead Coding PVM design Issue

Analysis: P-DTSTEP was taken from R-DTSTEP and reduced. Some sections of the remaining code are no necessary and can be removed, simplified, and/or corrected. In version 2.4.1.2 P-DTSTEP, the statement "if ( $\mathrm{dt}>\mathrm{dt}$ _min(i))go to 70" statement is always taken; so the coding thereafter to statement 70 can never be accessed and was removed. Moreover, in R-DTSTEP, $d t$ is halved if SUCCES is non-zero, and this test divides between minimal and non-minimal time-step handling. However, halving DT is not done in P-DTSTEP and so DT is always greater than the user input minimum at that point in the calculation.

Solution: The coding beneath it was corrected to write proper error messages, not adjust the time or time-step, not reset PVM_SUCCES, and jump to the 8001-message section of coding.

PVM0910 Stretch Logic Error Corrected $\quad$ PVM implementation error

Analysis: For a couple synchronously coupled problems, R-DTSTEP recognizes a stretch unusual time, calculates half the distance to the unusual time as time step, takes it, but on the next advancement, takes a shorter time step that reaches the next multiple of DTMAX. It thus ignored the stretch logic on the second step of the stretch handler logic. The Executive and child take different time steps and the coupled process hangs.

Solution: in R-DTSTEP

- In dthyCalc: DTHY $\rightarrow$ DTMAX_I

- $\quad$ In Unusual Handler: EDITTIME $->$ CURTMIN = min (endlnterval(i), curtxy) where $x y \varepsilon\{m i$, $\mathrm{mj}, \mathrm{rs}, \mathrm{ex \}}$. 


\title{
Implementation of a New DTSTEP Algorithm for use in RELAP5-3D and PVMEXEC
}

\author{
Completion Report
}

Solution: in P-DTSTEP

- Reset all curtxy if |endtime - curtxy $\mid<$ dtsmallest

PVM0911 PVMEXEC Input Error Messages $\quad$ PVM implementation deficiency

Analysis: Compared to RELAP5-3D, PVMEXEC provides little information to users about input errors. In fact under some circumstances, it will fail input with no message at all, such as if a blank line occurs after the executive's final timecard.

Solution (partial): A dozen new error messages were added to PVMEXEC in the subroutines INPUTD and RNEWP.

PVM0912 64-bit error in version 2.4.4 Script and source code errors

All PVM installation test cases failed in 64-bit installation mode.

Analysis: PVM Executive declared WAIT to be real*4, but describing it as real*8 in the PVM message because of an error in dpvmexec, the installation script. Also, ETIME was not declared REAL*4 in TIMSET in the envrl folder.

Solution: The script was corrected and the variable was declared properly.

PVM1001 Coupled Code Induced Infinite Loop New Coupling-only Error

Synchronously coupled codes enter an infinite loop caused by one code forcing a timestep repeat which causes the other to force timestep repeat in an unending cycle; see Figure 6.

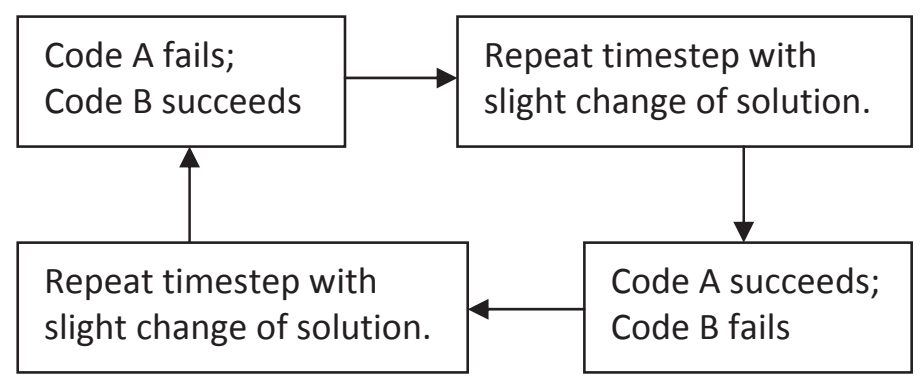

Figure 6. PVM3WAY Nodalization Diagram.

Analysis: This can occur when two or more codes each detect a failed timestep size that does not require a reduction in timestep size to clear. For RELAP5-3D, air appearance and velocity flip-flop are two mechanism that cause timestep repeat with same timestep size and slightly different conditions. The slightly different conditions then cause another coupled code to fail the advancement without requiring a timestep size reduction. If this failure results in a return to the conditions that caused the first code to fail, an infinite loop occurs; see Figure 6. Neither code can detect this condition itself.

Solution: Since the coupled analysis codes cannot detect this condition, code was implemented in PVMEXEC to detect the scenario. If two-successive timesteps have been failed at the same timestep size, PVMEXEC changes the timestep status from "pvm_samedt" to "pvm_smallerdt." Further, the original algorithm, which never ensured that a smaller timestep size was used if a status of "pvm_smallerdt" was received, was upgraded so whenever the "pvm_smallerdt" status is received from any of the codes, the largest timestep size that is allowed on the repeated timestep is $1 / 2$ of the previous timestep size. 


\section{Implementation of a New DTSTEP Algorithm for use in RELAP5-3D and PVMEXEC \\ Completion Report}

\subsection{Conclusions}

A new integer-based timestep selection and advancement algorithm has been implemented in the PVMEXEC and RELAP5-3D computer programs. A comprehensive code test suite was developed. The algorithm currently has no known problems associated with it. The work to develop the new algorithm was completed in time to support the specific need dates for a robust algorithm.

\subsection{Acknowledgements}

The work on this project was supported by consultants Dr. Walter Weaver and Hope Forsmann. Walt was a seminal author of the PVM coupling and provided explanations of many subtleties of the workings of the coupling as well as detailed insight into the coding. Hope was steadfast and instrumental in tracking down several of the errors listed in Section 11.

Several staff members at funding source were involved with the debugging and solving of user problems, creation of solutions to those problems, and their implementation of those solutions in the source code.

\subsection{References}

(a) Weaver, W. L., Tomlinson, E. T., Aumiller, D. L., 2002, “A PVM Executive Program for Use with RELAP5-3D," Proceedings of ICONE-10, Arlington VA, April 2002.

(b) W. L. Weaver, "Programmers Manual for the PVM Coupling Interface in the RELAP5-3D(C) Code," INL/EXT-05-00203, March 2005.

(c) A. R. Edwards and F. P. O'Brien, "Studies of Phenomena Connected with the Depressurization of Water Reactors," Journal of the British Nuclear Energy Society, Vol. 9, 1970, pp. 125-135.

(d) H. Christensen, "Power-to-Void Transfer Function," ANL-6385, 1961. 
Completion Report

\section{Appendix A}

Table 20 - Sample Testing Checklist

\begin{tabular}{|l|l|}
\hline \multicolumn{2}{|c|}{ RELAP5-3D data } \\
\begin{tabular}{|l|l|}
\hline $\begin{array}{l}\text { Location } \\
\text { of } \\
\text { executable }\end{array}$ & /mount/betp_p18/relap5.x \\
\hline $\begin{array}{l}\text { Compile } \\
\text { Date }\end{array}$ & 20100514 \\
\hline $\begin{array}{l}\text { Compile } \\
\text { Time }\end{array}$ & $12: 32: 04$ \\
\hline $\begin{array}{l}\text { Location of } \\
\text { executable }\end{array}$ & /mount/betp_p18/pvmexec.x \\
\hline $\begin{array}{l}\text { Compile } \\
\text { Date }\end{array}$ & PVMEXEC data \\
\hline $\begin{array}{l}\text { Compile } \\
\text { Time }\end{array}$ & $09: 41: 24$ \\
\hline
\end{tabular}
\end{tabular}

Testing Status

\begin{tabular}{|l|l|l|}
\hline Problem Name & Status & Comments \\
\hline \hline Matrices & Works & \\
\hline verify & Works & \\
\hline misc_cpl & Works & \\
\hline cobra_restart (enhanced) & Works & \\
\hline R5_melcor_hstr & Works & \\
\hline long_running_single_R5 & Works & \\
\hline User1 & Works & \\
\hline User2 & Works & \\
\hline User3 & Works & \\
\hline User4 & Works & \\
\hline User5 & Works & \\
\hline R5_melcor-ss_complete & Works & \\
\hline R5_melcor-tran1 & Works & \\
\hline
\end{tabular}

